\title{
Rat Organic Cation Transporter 1 Contains Three Binding Sites for Substrate 1-Methyl-4-phenylpyridinium per Monomer ${ }^{\text {[ }}$
}

\author{
Thorsten Keller, Valentin Gorboulev, Thomas D. Mueller, Volker Dötsch, Frank Bernhard, \\ and Hermann Koepsell
}

Institute of Anatomy and Cell Biology (T.K., V.G., H.K.) and Department of Molecular Plant Physiology and Biophysics, Juliusvon-Sachs-Institute (T.K., T.D.M., H.K.), University of Würzburg, Würzburg, Germany; and Department of Biophysical Chemistry, University of Frankfurt, Frankfurt am Main, Germany (V.D., F.B.)

Received June 29, 2018; accepted November 5, 2018

\section{ABSTRACT}

Organic cation transporters OCT1 (SLC22A1) and OCT2 (SLC22A2) are critically involved in absorption and excretion of diverse cationic drugs. Because drug-drug interactions at these transporters may induce adverse drug effects in patients, in vitro testing during drug development for interaction with the human transporters is mandatory. Recent data performed with rat OCT1 (rOCT1) suggest that currently performed in vitro tests assuming one polyspecific binding site are insufficient. Here we measured the binding and transport of model substrate 1-methyl-4-phenylpyridinium ${ }^{+}\left(\mathrm{MPP}^{+}\right)$by cell-free-expressed fusion proteins of rOCT1 and rOCT1 mutants with green fluorescent protein that had been reconstituted into nanodiscs or proteoliposomes. The nanodiscs were formed with major scaffold protein (MSP) and different phospholipids, whereas the proteoliposomes were formed with a mixture of cholesterol, phosphatidylserine, and phosphatidylcholine. In nanodiscs formed with 1-palmitoyl-2-oleoyl-sn-glycero-3-phosphocholine or cholesterol, phosphatidylserine, and phosphatidylcholine, two low-affinity $\mathrm{MPP}^{+}$binding sites and one high-affinity $\mathrm{MPP}^{+}$binding site per transporter monomer were determined. Mutagenesis revealed that tryptophan 218 and aspartate 475 in neighboring positions in the modeled outward-open cleft contribute to one low-affinity binding site, whereas arginine 440 located distantly in the cleft is critical for $\mathrm{MPP}^{+}$binding to another low-affinity site. Comparing $\mathrm{MPP}^{+}$binding with $\mathrm{MPP}^{+}$transport suggests that the low-affinity sites are involved in $\mathrm{MPP}^{+}$transport, whereas high-affinity $\mathrm{MPP}^{+}$binding influences transport allosterically. The data will be helpful in the interpretation of future crystal structures and provides a rationale for future in vitro testing that is more sophisticated and reliable, leading to the generation of pharmacophore models with high predictive power.

\section{Introduction}

The polyspecific organic cation transporters OCT1 (SLC22A1), OCT2 (SLC22A2), and OCT3 (SLC22A3) play a pivotal role in absorption, tissue distribution and elimination of cationic drugs including psychopharmaca, cytostatics, antidiabetics, and antiviral drugs (Amphoux et al., 2006; Koepsell et al., 2007; Minuesa et al., 2011; Nies et al., 2011). These transporters also have important physiologic functions because they translocate various endogenous cations. To anticipate drug-drug interactions at the level of individual organic cation transporters (OCTs), in vitro evaluation of novel drugs for interaction with human OCT1 (hOCT1) and

This work was supported by the Deutsche Forschungsgemeinschaft (KO 862/6-1).

https://doi.org/10.1124/mol.118.113498.

S This article has supplemental material available at molpharm. aspetjournals.org.
OCT2 (hOCT2) has been recommended (Giacomini et al., 2010, Zamek-Gliszczynski et al., 2018) and is demanded by the American Food and Drug Administration (https://www. fda.gov/downloads/Drugs/GuidanceComplianceRegulatoryInformation/Guidance/UCM581965.pdf) and the European Medicine Agencies (http://www.ema.europa.eu/en/docs/enGB/ documentlibrary/Scientificguideline/2012/07/WC500129606. pdf). Currently, novel molecular entities (NMEs) are tested for interaction with hOCT1 and hOCT2 expressed in epithelial cells to determine whether they inhibit uptake of a cationic model substrate that is applied at a micromolar concentration (Ahlin et al., 2008, 2011; Chen et al., 2017). This procedure has turned out to be insufficient because it was observed that the efficacy of inhibitors was dependent on the molecular structure of the employed substrate and was different when substrate concentrations far below their respective Michaelis-Menten constant $\left(K_{\mathrm{m}}\right)$ values were used for uptake measurements (Belzer et al., 2013; Thévenod et al., 2013;

ABBREVIATIONS: 3D, three-dimensional; ANOVA, analysis of variance; $B_{\max }$, maximal binding; CHAPS, 3-[(3-cholamidopropyl)dimethylammonio]-1propanesulfonate; DMPG, 1,2-dimyristoyl-sn-[phospho-rac-(1-glycerol)]; DPPC, 1,2-dipalmitoyl-sn-glycero-3-phosphocholine; EC $\mathrm{E}_{50}$, half-maximal effective concentration; GFP, green fluorescent protein; HEK, human embryonic kidney; hOCT, human OCT; $K_{m}$, Michelis-Menten constant; MFS,

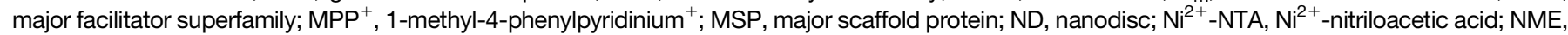
novel molecular entity; OCT, organic cation transporter; PBS, phosphate buffered saline; PC, 1,2-diacyl-sn-glycero-3-phospho-L-serine; POPC, 1palmitoyl-2-oleoyl-sn-glycero-3-phosphocholine; PS, 1,2-diacyl-sn-glycero-3-phospho-L-serine; rOCT, rat organic cation transporter; TBuA ${ }^{+}$, tetrabutylammonium ${ }^{+}$. 
Yin et al., 2016; Minuesa et al., 2017; Gorboulev et al., 2018; Sandoval et al., 2018). For example, high-affinity inhibition of uptake of 1-methyl-4-phenylpyridinium ${ }^{+}\left(\mathrm{MPP}^{+}\right)$by hOCT1, hOCT2, and human OCT3 (hOCT3) by the nucleoside reverse transcriptase inhibitor tenofovir disoproxil fumarate was observed when uptake was measured with $12.5 \mathrm{nM} \mathrm{MPP}{ }^{+}$ but did not show up when uptake was performed with $1 \mu \mathrm{M}$ $\mathrm{MPP}^{+}$(Minuesa et al., 2009, 2017). Such observations indicate the necessity for more sophisticated in vitro testing of NMEs during drug development (Koepsell, 2018). To establish appropriate test protocols and to enforce their application by pharmaceutical companies, a basic experimentally supported concept of how different drugs can interact at individual OCTs is imperative.

The current knowledge about function and substrate recognition by OCTs is mainly derived from studies performed with rat OCT1 (rOCT1) and rat OCT2 (rOCT2). Evidence has been provided that rOCT1 and rOCT2 are facilitated diffusion systems that may operate as electrogenic cation uniporters or electroneutral cation exchangers (Busch et al., 1996; Budiman et al., 2000; Keller et al., 2005; Schmitt and Koepsell, 2005). The apparent $K_{\mathrm{m}}$ values of OCTs for different substrates are in the micromolar and millimolar concentration range. In addition, high-affinity cation interaction sites with half-maximal effective concentrations $\left(\mathrm{EC}_{50}\right)$ in the picomolar and nanomolar ranges have been determined and may be inhibitory (Gorbunov et al., 2008; Minuesa et al., 2009). Homooligomerization of OCT1 and OCT2 has been demonstrated (Keller et al., 2011; Brast et al., 2012), however, it remained unclear whether high-affinity binding is dependent on oligomerization. To obtain molecular insight into polyspecific binding and transport, extensive mutagenesis was performed in rOCT1. Effects on apparent $K_{\mathrm{m}}$ values and on half-maximal concentration values of inhibitors were interpreted with the help of homology models derived from crystal structures of bacterial transporters of the same major facilitator superfamily (MFS) (Gorboulev et al., 1999, 2005, 2018; Popp et al., 2005; Volk et al., 2009; Egenberger et al., 2012). The data support the hypothesis that rOCT1 contains clefts with cation binding domains in the outward- or inward-open conformation, which contain overlapping binding sites for structurally different cations. However, these interpretations remained speculative because changes in $K_{\mathrm{m}}$ and $I C_{50}$ values observed upon mutagenesis do not directly reflect effects on binding of substrates and/or inhibitors.

In the present study we measured the binding of radioactively labeled 1-methyl-4-phenylpyridinium ${ }^{+}\left(\mathrm{MPP}^{+}\right)$to cellfree-expressed rOCT1 and rOCT1 mutants fused with green fluorscent protein (GFP) that had been reconstituted into nanodiscs (NDs). We analyzed apparent dissociation constant $\left(K_{\mathrm{D}}\right)$ values for $\mathrm{MPP}^{+}$binding to reconstituted NDs and the amounts of $\mathrm{MPP}^{+}$per rOCT1 monomer in the preparation. In addition, we reconstituted the proteins into proteoliposomes and determined the effects of the mutations on the apparent $K_{\mathrm{m}}$ values and the turnover numbers. We observed that each rOCT1 monomer contained two low-affinity $\mathrm{MPP}^{+}$binding sites that are directly engaged in transport and one highaffinity $\mathrm{MPP}^{+}$binding site that exhibits allosteric effects on the low-affinity sites. These data provide a basis for understanding interactions of cationic ligands at the highly polyspecific organic cation transporters.

\section{Materials and Methods}

Materials. $\left[{ }^{3} \mathrm{H}\right]-1$-methyl-4-phenylpyridinium ${ }^{+}\left(\mathrm{MPP}^{+}\right)(3.1$ $\mathrm{TBq} / \mathrm{mmol}$ ) was obtained from ART Chemicals (Amsterdam, Holland). 1-Myristoyl-2-hydroxy-sn-glycero-3(phospho-rac-(1-glycerol)) (cat. no. 858120), 1,2-dimyristoyl-sn-glycero-3-phospho-(1-rac-glycerol) (DMPG) (cat. no. 840445P), 1,2-dipalmitoyl-sn-glycero-3-phosphocholine (DPPC) (cat. no. 850355P), and 1-palmitoyl-2-oleoyl-sn-glycero-3-phosphocholine (POPC) (cat. no. 850457P) were purchased from Avanti Polar Lipids (Alabaster, AL). Cholesterol (cat. no. C8667), 1,2-diacyl-sn-glycero-3phosphocholine from egg yolk (PC) (cat. no. P3556), 1,2-diacyl-snglycero-3-phospho-L-serine from bovine brain (PS) (cat. no. P7769), and goat anti-rabbit IgG antibody coupled to horse radish peroxidase (cat. no.12-348) were delivered by Sigma-Aldrich (Schnelldorf, Germany). Dodecyl-phosphocholine (cat. no. F308S) was obtained from Anatrace (Maumee, $\mathrm{OH})$ and 3-[(3-cholamidopropyl)dimethylammonio]-1-propanesulfonate (CHAPS) (cat. no. A1099) from AppliChem GmbH (Darmstadt, Germany). Escherichia coli strain BL21 Star(D3) (cat. no. C6010-03) was purchased from Invitrogen/Thermo Fisher Scientific (Carlsbad, CA) and E. coli strain A19 (cat. no.5997) from Coli Genetic Stock Center (New Haven, CT). Plasmid EGFP-C1 (cat. no. 6084-1) was obtained from Takara Bio Inc. (Kusatsu, Japan), plasmid pIVEX2.3-MCS (cat. no. 3253 538) from Roche Diagnostics (Mannheim, Germany), plasmid pET21a (cat. no. 69740-3) from Merck Life Science GmbH (Eppelheim, Germany), and plasmid pcDNA3.1 (cat. no. V790-20) from Invitrogen/Thermo Fisher Scientific. Other materials were obtained as described (Keller et al., 2008).

Cloning. For cloning of the fusion proteins, we employed the GFP mutant A206K, which does not form dimers (Zacharias et al., 2002). The GFP mutant A206K was created by polymerase chain reaction using EGFP-C1 plasmid as template. A forward flanking primer with the NdeI recognition site and a reverse flanking primer with the BamHI recognition sequence followed by an XhoI site were employed. The amplificate was cut with $\mathrm{NdeI} / \mathrm{XhoI}$ and cloned into the respective sites of the pIVEX2.3-MCS vector.

To generate GFP(A206K)-rOCT1 with a His-tag at the C-terminus, GFP(A206K) cDNA was cloned upstream of rOCT1 into NdeI/BamHI sites of the rOCT1-His/pET21a plasmid (Keller et al., 2011). The resulting plasmid was cut with $\mathrm{NdeI} / \mathrm{XhoI}$, and the cDNA of GFP (A206K)-rOCT1 was transferred into the $\mathrm{NdeI} / \mathrm{XhoI}$ sites of the vector pIVEX2.3-MCS

Mutants GFP(A206K)-rOCT1(F160Y), GFP(A206K)-rOCT1(W218L), and GFP(A206K)-rOCT1(W218Y) were cloned by replacing the Eco147I/Eco81I fragment of the rOCT1 by the fragments of the respective rOCT1 mutants prepared previously (Popp et al., 2005; Volk et al., 2009).

Mutants GFP(A206K)-rOCT1(R440K), GFP(A206K)-rOCT1(L447Y), GFP(A206K)-rOCT1(L447F), GFP(A206K)-rOCT1(Q448E), and GFP (A206K)-rOCT1(D475E) were generated by replacing the Eco81I/XhoI fragment of the rOCT1 by the respective fragments of rOCT1 mutants described earlier (Gorboulev et al., 1999, 2005; Volk et al., 2009). To construct the rOCT1 double mutant GFP(A206K)-rOCT1(W218Y/D475E), the Eco81I/XhoI fragment of GFP(A206K)-rOCT1(W218Y) was replaced by the respective fragment of the GFP(A206K)-rOCT1(D475E). The rOCT1 double mutant GFP(A206K)-rOCT1(R440K/D475E) was made by polymerase chain reaction with the GFP(A206K)-rOCT1(D475E) as a template.

For cloning of GFP(A206K)-rOCT1 $(6 \Delta \mathrm{C})$ the pIVEX2.3-MCS vector was used after removal of the unique BamHI site. Therefore, the plasmid was linearized with BamHI, the ends were filled using DNA polymerase I, and the plasmid was ligated. The GFP(A206K)-rOCT1 cDNA was cloned first into the NdeI/XhoI sites of this vector. Then the BamHI/Eco81I fragment of the rOCT1 WT was replaced by the respective fragment of the $\operatorname{rOCT} 1(6 \Delta \mathrm{C})$, which had been prepared from the previously described GFP-rOCT1 $(6 \Delta \mathrm{C}) / \mathrm{pcDNA} 3.1$ plasmid (Keller et al., 2011).

Expression and Purification of Major Scaffold Protein 1E3D1. A His-tagged elongated construct of membrane scaffold 
protein 1 which contains a truncated first $\alpha$-helix (MSP1E3D1) (Denisov et al., 2004; Roos et al., 2012) was expressed in E. coli. Bacteria ( $E$. coli strain BL21(DE3)Star) were transformed with pET28a plasmid encoding His-tagged MSP1E3D1 and grown to midlog phase. Protein expression was induced by isopropyl- $\beta$-D-thiogalactopyranoside and bacteria were grown for 3 hours at $30^{\circ} \mathrm{C}$. After a 15 -minute centrifugation at $6000 \mathrm{~g}$, bacteria were washed, suspended in $20 \mathrm{mM}$ Tris- $\mathrm{HCl} \mathrm{pH} 8.0$ containing $500 \mathrm{mM} \mathrm{NaCl}$ and $50 \mathrm{mM}$ imidazole, lysed by sonication at $4^{\circ} \mathrm{C}$, and cellular debris was removed by a 1 -hour centrifugation at $100,000 \mathrm{~g}$. For protein purification, the supernatant was mixed with $\mathrm{Ni}^{2+}$-nitriloacetic acid $\left(\mathrm{Ni}^{2+}\right.$-NTA)agarose, incubated for 1 hour under rotation, and poured into an empty gravity flow column. After extensive washing with $20 \mathrm{mM}$ Tris$\mathrm{HCl} \mathrm{pH} 8.0$ containing $500 \mathrm{mM} \mathrm{NaCl}$ and $50 \mathrm{mM}$ imidazole, protein was eluted with the same buffer containing $500 \mathrm{mM}$ imidazole. Fractions containing purified protein were pooled. Aliquots were snap frozen in liquid nitrogen and stored at $-80^{\circ} \mathrm{C}$.

Formation of Empty Nanodiscs. The following were incubated for 1 hour at room temperature: $0.1 \%(\mathrm{w} / \mathrm{v})$ dodecyl-phosphocholine containing $20 \mu \mathrm{M}$ MSP1E3D1 plus either $2.2 \mathrm{mM}$ DMPG or $2.2 \mathrm{mM}$ DPPC or $2.2 \mathrm{mM}$ POPC or $0.6 \mathrm{mg} / \mathrm{ml}$ cholesterol, $0.2 \mathrm{mg} / \mathrm{ml}$ 1,2-diacylsn-glycero-3-phosphocholine (PC) and $1 \mathrm{mg} / \mathrm{ml}$ 1,2-diacyl-sn-glycero-3phospho-L-serine (PS). For nanodisc formation, the mixture was dialyzed for 48 hours at $0^{\circ} \mathrm{C}$ against excess of dialysis buffer $(40 \mathrm{mM}$ Tris-HCl pH 8.0, $100 \mathrm{mM} \mathrm{NaCl)} \mathrm{employing} \mathrm{three} \mathrm{buffer} \mathrm{changes.}$ Residual aggregates were separated from soluble NDs by a 20 -minute centrifugation at $22,000 \mathrm{~g}$. The supernatant was transferred to Centriprep concentrator devices (10-kDa MWCO; Millipore, Merck, Darmstadt, Germany). After equilibration with $3 \mathrm{ml}$ of dialysis buffer, the concentrator devices were centrifuged at $2000 \mathrm{~g}\left(4^{\circ} \mathrm{C}\right)$ and NDs were concentrated up to final MSP1E3D1 concentrations of $2.4 \mathrm{mM}$ corresponding to a concentration of NDs of $1.2 \mathrm{mM}$. After another 20-minute centrifugation at 22,000g, the NDs in the supernatant were kept on ice before usage. For long-term storage, NDs were snap-frozen in liquid nitrogen and stored at $-80^{\circ} \mathrm{C}$.

Cell-Free Expression and Insertion into Nanodises. Extracts for cell-free expression were isolated from E. coli strain A19 and reaction mixtures containing vectors and cell-free extracts as well as feeding mixtures were prepared as described (Klammt et al., 2004). Cell-free expression of GFP-rOCT1 wild-type and mutants and cotranslational insertion into nanodiscs was performed in the absence of detergent in the continuous exchange mode. Dispodialysers from Spectrum Laboratories Inc. (Breda, Netherlands) with a cutoff of $25 \mathrm{kDa}$ and a 1-ml reaction volume were used. For cotranslational membrane insertion of the transport proteins during cell-free synthesis, $150 \mu \mathrm{M}$ empty NDs were added to the reaction mixtures. Cell-free reaction was performed by shaking the Dispodialysers filled with reaction mixtures for 20 hours at $30^{\circ} \mathrm{C}$ in glass tubes containing $17 \mathrm{ml}$ of feeding mixture. Thereafter, the reaction mixture was centrifuged for 10 minutes at $10,000 \mathrm{~g}\left(4^{\circ} \mathrm{C}\right)$ and supernatant containing empty NDs and the NDs with reconstituted transport proteins was harvested. For binding measurements, $\mathrm{Ni}^{2+}$-NTA-agarose beads were added to the supernatant and the beads with attached NDs were precipitated.

Measurement of MPP $^{+}$-Binding. NDs attached to $\mathrm{Ni}^{2+}$-NTAagarose beads were incubated for 1 minute at $37^{\circ} \mathrm{C}$ or $0^{\circ} \mathrm{C}$ with phosphate buffered saline (PBS) $\mathrm{pH} 7.4$ containing $12.5 \mathrm{nM} \mathrm{MPP}{ }^{+}$ traced with $\left[{ }^{3} \mathrm{H}\right] \mathrm{MPP}^{+}$without and with different additional concentrations of nonradioactive $\mathrm{MPP}^{+}$. The samples were washed for about 10 seconds with $5 \mathrm{ml}$ of ice-cold PBS on $0.22-\mu \mathrm{m}$ cellulose acetate filters. The filters were dissolved in LUMASAFE PLUS mixture (Lumac LSC, Groningen, The Netherlands) and assayed for radioactivity by liquid scintillation counting. A one-minute incubation with $\mathrm{MPP}^{+}$at $37^{\circ} \mathrm{C}$ was shown to be long enough to achieve saturation of binding at high- and low-affinity $\mathrm{MPP}^{+}$binding sites of GFP-rOCT1 (Supplemental Fig. 1, left panel). Since MPP ${ }^{+}$binding to GFP-rOCT1 was not decreased when washing of the filters was extended using 10 or $15 \mathrm{ml}$ of washing buffer, a dissociation of bound $\mathrm{MPP}^{+}$during routine washing with $5 \mathrm{ml}$ was considered negligible (Supplemental Fig. 1, right panel). Because the slow dissociation of $\mathrm{MPP}^{+}$could have been attributable to a structural change in the transporter, possibly induced by $\mathrm{MPP}^{+}$binding and/or by cooling during the washing procedure, the determined dissociation constants were considered as apparent dissociation constants.

Purification of Cell-Free Expressed Transporters. Cell-free expression was performed in the absence of detergent without addition of NDs as described above. The pellet obtained after a 10-minute centrifugation at $10,000 \mathrm{~g}\left(4^{\circ} \mathrm{C}\right)$ was washed with Tris buffer $(20 \mathrm{mM}$ Tris- $\mathrm{HCl} \mathrm{pH} \mathrm{8,500} \mathrm{mM} \mathrm{NaCl})$ containing $10 \mathrm{mM}$ imidazole. The precipitate was solubilized by a 1 -hour incubation at $30^{\circ} \mathrm{C}$ with $1 \mathrm{ml}$ of $2 \%$ (w/v) 1-myristoyl-2-hydroxy-sn-glycero-3(phospho-rac-(1-glycerol)) dissolved in Tris buffer containing $10 \mathrm{mM}$ imidazole and centrifuged for 10 minutes at $10,000 \mathrm{~g}\left(4^{\circ} \mathrm{C}\right)$. The supernatant was mixed with $11 \mathrm{ml}$ of Tris buffer containing $10 \mathrm{mM}$ imidazole plus $1 \%(\mathrm{w} / \mathrm{v})$ CHAPS and $\mathrm{Ni}^{2+}$-NTA-agarose and incubated for 1 hour at $4^{\circ} \mathrm{C}$. The suspension was poured into a column and washed with Tris buffer containing $1 \%$ CHAPS and $10 \mathrm{mM}$ imidazole and Tris buffer containing 1\% CHAPS and $20 \mathrm{mM}$ imidazole. Proteins were eluted with $5 \mathrm{ml}$ of Tris buffer containing 1\% CHAPS and $100 \mathrm{mM}$ imidazole.

Reconstitution of Proteoliposomes. For freeze-thaw reconstitution protein-lipid aggregates were mixed with large multilamellar liposomes, and the mixture was frozen, thawed, pelleted, and homogenized as described (Keller et al., 2011). Protein-lipid aggregates were formed as follows: Cholesterol, PC, and PS (1 mg of each) were solubilized in $1 \mathrm{ml}$ of chloroform/methanol (1:1, v/v), dried in a round-bottom flask under nitrogen, and $500 \mu \mathrm{l}$ of the purified protein solved in CHAPS was added. The mixture was shaken for 1 hour at $4^{\circ} \mathrm{C}$, and CHAPS was removed by dialysis at $4^{\circ} \mathrm{C}$ against $20 \mathrm{mM}$ Tris/ $\mathrm{HCl}, 500 \mathrm{mM} \mathrm{NaCl}$, and $100 \mathrm{mM}$ choline chloride. After a 30 -minute centrifugation at $200,000 \mathrm{~g}\left(4^{\circ} \mathrm{C}\right)$, the sediment was suspended in $2 \mathrm{ml}$ of ice-cold $\mathrm{KC}$ buffer $(20 \mathrm{mM}$ imidazole $\mathrm{pH} 7.4,0.1 \mathrm{mM}$ $\mathrm{Mg}^{2+}, 100 \mathrm{mM} \mathrm{K}{ }^{+}, 100 \mathrm{mM}$ cyclamate ${ }^{-}$), again centrifuged at $200,000 \mathrm{~g}$, and the pellet was suspended in $150 \mu \mathrm{l}$ of KC buffer. Large multilamellar liposomes were prepared in the following way: $2 \mathrm{mg}$ of cholesterol and $4 \mathrm{mg}$ of PS in $1 \mathrm{ml}$ of chloroform/methanol (1:1, v/v) were dried in a round-bottom flask under nitrogen, $1 \mathrm{ml}$ of $\mathrm{KC}$ buffer was added, and the flask was shaken for 3 hours at room temperature. Aggregates were removed by a 10 -minute centrifugation at $10,000 \mathrm{~g}$. The multilamellar liposomes in the supernatant were pelleted by a 15-minute centrifugation at $200,000 \mathrm{~g}$ and suspended in $150 \mu \mathrm{l}$ of $\mathrm{KC}$ buffer (room temperature). For the formation of large proteoliposomes $150 \mu \mathrm{l}$ of the protein-lipid aggregates were mixed with $150 \mu \mathrm{l}$ of the multilamellar liposomes, incubated for 15 minutes at $41^{\circ} \mathrm{C}$, and frozen in liquid nitrogen. Before transport measurements were started, the sample was thawed at $37^{\circ} \mathrm{C}$ in a water bath, $1.7 \mathrm{ml} \mathrm{KC}$ buffer (room temperature) was added, the proteoliposomes were pelleted by a 15-minute centrifugation at room temperature, the pellet was resuspended in $300 \mu \mathrm{l} \mathrm{KC}$ buffer (room temperature), and homogenized by repeated suction into and forced extrusion out of a $100-\mu$ l pipette tip.

Measurement of MPP $^{+}$-Uptake into Proteoliposomes. The proteoliposomes were preincubated for 10 minutes at $37^{\circ} \mathrm{C}$ with $20 \mu \mathrm{M}$ valinomycin in the absence or presence of $100 \mu \mathrm{M}$ quinine. Quinine is an established high-affinity inhibitor of rOCT1-mediated transport (Koepsell et al., 2007). It inhibits rOCT1-mediated transport of $12.5 \mu \mathrm{M} \mathrm{MPP}^{+}$into proteoliposomes to a degree similar to other high-affinity inhibitors of rOCT1 such as cyanine863 and tetrapentylammonium $^{+}$(Keller et al., 2005). One-second uptake of $\mathrm{MPP}^{+}$was measured at $37^{\circ} \mathrm{C}$ in the absence and presence of quinine. Ten microliters of prewarmed proteoliposomes $\left(37^{\circ} \mathrm{C}\right)$ were mixed with $90 \mu \mathrm{l}$ of prewarmed $\left(37^{\circ} \mathrm{C}\right)$ sodium cyclamate buffer $(20 \mathrm{mM}$ imidazole $\mathrm{pH}$ 7.4, 0.1 $\mu \mathrm{M} \mathrm{Mg}^{2+}, 100 \mathrm{mM} \mathrm{Na}^{+}, 100 \mathrm{mM}$ cyclamate ${ }^{-}$), which contained different concentrations of $\mathrm{MPP}^{+}$that were traced with $\left[{ }^{3} \mathrm{H}\right] \mathrm{MPP}^{+}$. Proteoliposomes pretreated with quinine were incubated in the presence of $100 \mu \mathrm{M}$ quinine. One-second incubation was performed as follows: The proteoliposomes were placed at the inner wall of the reaction tube 
just above the incubation medium. Uptake was initiated by mixing on a switched-on vortexer and stopped after 1 second by adding ice-cold stop solution (KC buffer containing $100 \mu \mathrm{M}$ quinine) via a prepositioned pipette. A metronome was used to perform the 1-second incubation measurements. Radioactivity was determined by washing the proteoliposomes on $0.22-\mu \mathrm{m}$ cellulose acetate filters and liquid scintillation counting of the solubilized filters. $\mathrm{MPP}^{+}$uptake was corrected for $\mathrm{MPP}^{+}$ uptake measured in the presence of quinine.

SDS-PAGE and Western Blotting. Samples were incubated for 30 minutes at $37^{\circ} \mathrm{C}$ in $60 \mathrm{mM}$ Tris- $\mathrm{HCl} \mathrm{pH} 6.8$, containing $2 \%(\mathrm{w} / \mathrm{v})$ SDS, 7\% (v/v) glycerol, and $100 \mathrm{mM}$ dithiothreitol. SDS-PAGE, staining of gels with Coomassie brilliant blue, and electroblotting to a polyvinylidene difluoride membrane were performed as described previously (Keller et al., 2008). For immunostaining of GFP-rOCT1 or mutants of GFP-rOCT1, an antibody against the large extracellular loop of rOCT1 raised in rabbit and goat anti-rabbit IgG coupled to horseradish peroxidase were used (Meyer-Wentrup et al., 1998). Binding of horseradish peroxidase-coupled IgG was visualized using enhanced chemiluminescence (ECL system; Amersham Buchler, Braunschweig, Germany). Prestained molecular weight markers (BenchMark; Invitrogen) were used to determine apparent molecular masses.

Estimation of Transporter in Nanodiscs. In NDs coupled to $\mathrm{Ni}^{+}$-NTA-agarose beads that were used for $\mathrm{MPP}^{+}$binding measurements the concentrations of GFP-rOCT1 wild-type or GFP-rOCT1 mutants were determined by analysis of total protein $\left(\mathrm{P}_{\text {total }}\right)$ in combination with densitometry of Coomassie-stained SDS polyacrylamide gels. Since the preparation consisted of empty NDs that contained only MSP1E3D1 protein or loaded NDs that contained only MSP1E3D1 and GFP-rOCT1 wild-type or one of the GFP-rOCT1 mutants (see Fig. 1B), the protein concentration of GFP-fusion $\left(\mathrm{P}_{\mathrm{GFP}-\mathrm{F}}\right)$ protein could be calculated as follows:

$$
\mathrm{P}_{\mathrm{GFP}-\mathrm{F}}=\mathrm{P}_{\mathrm{total}} \times \mathrm{S}_{\mathrm{GFP}-\mathrm{F}} /\left(\mathrm{S}_{\mathrm{MSP} 1 \mathrm{E} 3 \mathrm{D} 1}+\mathrm{S}_{\mathrm{GFP}-\mathrm{F}}\right)
$$

where $\mathrm{P}_{\text {total }}$ represents the total protein concentration; $\mathrm{S}_{\mathrm{GFP}-\mathrm{F}}$, staining of the respective GFP fusion protein; and $\mathrm{S}_{\mathrm{MSP1E3D} 1}$, staining of MSP1E3D1. Staining of proteins in Coomassie-stained gels was quantified by densitometry using the open access program ImageJ1 developed by the National Institutes of Health. It was verified that the staining intensities obtained for GFP-rOCT1 and MSP1E3D1 were correlated equally with protein concentrations (Supplemental Fig. 2).

In Silico Docking of $\mathrm{MPP}^{+}$to Models of rOCT1. In silico docking was performed to outward-facing and inward-facing homology models of rOCT1 (Popp et al., 2005; Volk et al., 2009). All docking simulations were performed using the software package SYBYL version 7.1 (Tripos Inc., St. Louis, MO) and employed the force field MMFF94s. A coordinate file of the organic cation $\mathrm{MPP}^{+}$was built in SYBYL assuming standard geometry deduced from its twodimensional SMILES (Simplified Molecular Input Line Entry System) description, hydrogen atoms were added to the initial coordinate set and the three-dimensional (3D) structure of $\mathrm{MPP}^{+}$was subsequently energy-minimized by a conjugate gradient algorithm. The models of rOCT1 in its inward-facing and outward-facing conformations were complemented with hydrogens, partial charges were assigned using the AMBER7 FF02 force field (SYBYL version 7.1), and the rOCT1 structures carrying all-hydrogens were subsequently energyminimized with a conjugate gradient algorithm and the positions of all heavy atoms restrained by a strong positional harmonic potential. Docking simulations of $\mathrm{MPP}^{+}$to both conformations of the rOCT1 model were performed using the FlexX module of the software SYBYL version 7.1. For the simulations, the side chains of lysine residues were protonated, the carboxylate groups of aspartic and glutamic acid residues were nonprotonated, and cysteine and histidine residues were treated as neutral. In a first round of docking simulations, any site in the interior of the inward-facing or outward-facing cleft was accepted and the results were sorted according to their docking energies using the corresponding total FlexX-Scores. Since many

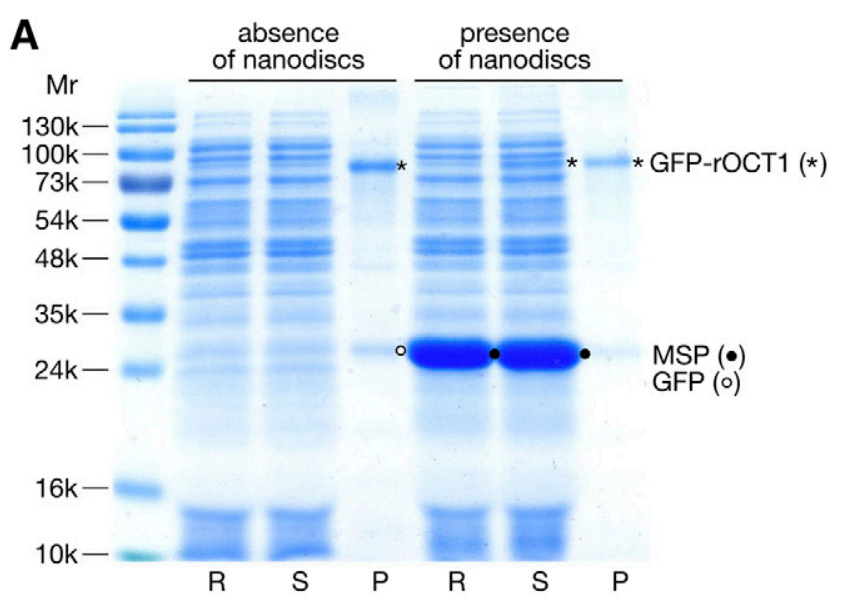

B

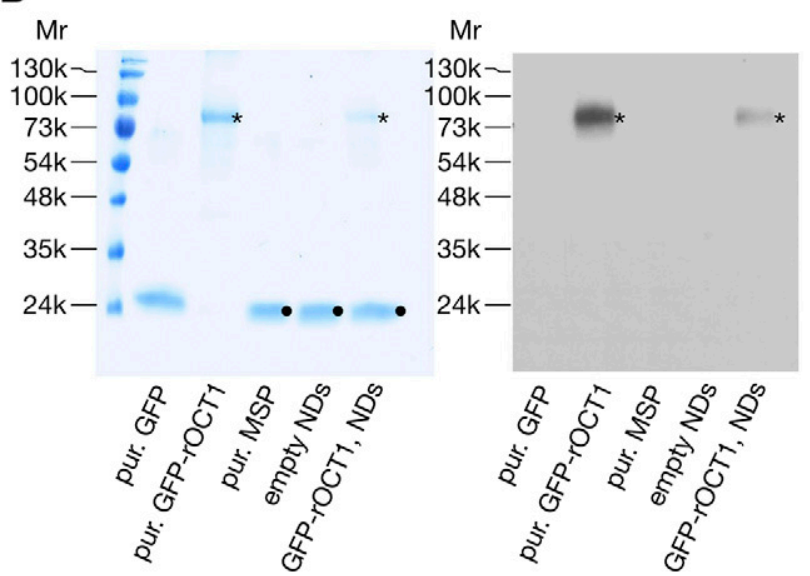

Fig. 1. Reconstitution of GFP-rOCT1 into nanodiscs formed with MSP1E3D1 and DMPG and their isolation. (A) Cell-free expression of Histagged GFP-rOCT1 in absence or presence of empty NDs composed of DMPG and His-tagged MSP1E3D1 (MSP). An SDS-polyacrylamide gel stained with Coomassie brilliant blue is shown. Lanes $\mathrm{R}$ show proteins in reaction mixtures without and with NDs at the beginning of cell-free synthesis. After completion of the synthesis, the reaction mixtures were centrifuged for 20 minutes at $22,000 \mathrm{~g}$. The supernatants are shown in lanes $\mathrm{S}$ and the pellets in lanes $\mathrm{P}$. When the synthesis was performed in the presence of NDs some GFP-rOCT1 appeared in the supernatant, whereas the amount of GFP-rOCT1 in the pellet was decreased. (B) Preparation of NDs containing His-tagged GFP-rOCT1 that was employed for $\mathrm{MPP}^{+}$ binding measurements. Using $\mathrm{Ni}^{2+}$-NTA linked to agarose beads, the Histagged NDs were precipitated from the $22,000 \mathrm{~g}$ supernatant obtained after cell-free expression of GFP-rOCT1 in the presence of NDs. Purified GFP (pur. GFP), purified GFP-rOCT1 (pur. GFP-rOCT1), purified MSP1E3D1 (pur. MSP), empty NDs (empty NDs), and precipitated NDs with reconstituted GFP-rOCT1 (GFP-rOCT1, NDs) were separated by SDSPAGE and stained with Coomassie brilliant blue (left panel) or visualized in a Western blot (right panel), which was developed with an antibody against rOCT1. Two micrograms of protein were applied per lane.

docked MPP molecules were found in close proximity to residues Trp218, Arg440, and Asp475, a second round of docking simulations was performed with the docking considered successful only when it occurred within a $7 \AA$ radius around these three residues. Again the results were sorted according to the docking energy and the top 30 results were analyzed.

Statistics. To determine the concentration dependence of $\mathrm{MPP}^{+}$ binding to GFP-rOCT1 wild-type or GFP-rOCT1 mutants reconstituted into NDs or transporter-mediated $\mathrm{MPP}^{+}$uptake into proteoliposomes, three independent experiments were performed in which 8-11 different $\mathrm{MPP}^{+}$concentrations were analyzed and three parallel measurements were performed per experimental condition. A one-site or two-site 
binding model was fitted to the binding data and the Michaelis-Menten equation to the uptake data of individual experiments. Mean values \pm S.D. of three experiments are presented. The software package GraphPad Prism Version 4.1 (GraphPad Software, LaJolla, CA) was used to compute statistical parameters. When more than two groups were compared, the statistical significance of differences was determined by one-way analysis of variance (ANOVA) with post-hoc Dunnett comparison (effects of mutants on binding or transport) or by two-way ANOVA with Bonferroni correction (comparison between effects of mutants on binding vs. transport). When two individual groups were compared, the Student's $t$ test was used. $P<0.05$ was considered statistically significant. The curves presented in the figures were obtained by fitting the one-site or two-site model or the Michaelis-Menten equation to the compiled data sets.

\section{Results}

Identification of Two MPP ${ }^{+}$Binding Sites per rOCT1 Monomer in Nanodises Formed with 1,2-Dimyristoylphosphoglycerol. For reconstitution of rOCT1 into NDs, we performed cell-free expression of His-tagged rOCT1 fusion protein with a nondimerizing mutant of green fluorescent protein (Zacharias et al., 2002) (GFP-rOCT1) in the absence of detergent and presence of empty NDs (Roos et al., 2013). The N-terminal fusion of rOCT1 with GFP did not impair the transport activity because the apparent $K_{\mathrm{m}}$ value for $\mathrm{MPP}^{+}$ uptake determined after reconstitution of GFP-rOCT1 into proteoliposomes was similar to the apparent $K_{\mathrm{m}}$ after reconstitution of purified rOCT1 (Keller et al., 2005, 2008). Empty NDs were formed by detergent removal from 1,2dimyristoyl-sn-[phospho-rac-(1-glycerol)] (DMPG) and Histagged membrane scaffold protein variant MSP1E3D1 that had been dissolved with dodecyl-phosphocholine (Denisov et al., 2004; Roos et al., 2012). When cell-free expression in the absence of detergent was performed without addition of NDs, GFP-rOCT1 and some free GFP were observed in a $22,000 g$ pellet (Fig. 1A, see left P). After cell-free expression in the presence of empty NDs, NDs with reconstituted GFPrOCT1 appeared in the $22,000 \mathrm{~g}$ supernatant, and the amount of GFP-rOCT1 in the 22,000g pellet was decreased (Fig. 1A, see $\mathrm{S}$ and $\mathrm{P}$ ). For binding measurements, the NDs in the supernatant were purified with $\mathrm{Ni}^{2+}{ }_{-}$NTA agarose beads (Fig. 1B, see GFP-rOCT1, NDs). This preparation contained two proteins, the nanodisc component MSP1E3D1 (see MSP in Fig. 1) and GFP-rOCT1. Densitometric analysis revealed that GFP-rOCT1 represented $1.8 \% \pm 0.3 \%(n=6)$ of the total protein (Supplemental Table 1). Assuming that each GFPrOCT1 containing nanodisc includes two MSP1E3D1 molecules and one GFP-rOCT1 monomer (Roos et al., 2013), about $1 \%$ of the NDs contained GFP-rOCT1. Considering the low percentage of NDs containing reconstituted transporter, the absence of post-translational modifications after cell-free expression, and the reconstitution of GFP-rOCT1 into NDs in the absence of detergent, it is probable that the NDs containing GFP-rOCT1 represent a homogeneous population.

For binding measurements, samples were incubated with radioactively labeled $\mathrm{MPP}^{+}$for 1 minute at $37^{\circ} \mathrm{C}$ and washed on cellulose acetate filters. Low nonspecific binding of MPP to filters, to agarose beads, to GFP linked to agarose beads, and to empty NDs linked to agarose beads was observed (Fig. 2A). In contrast, we observed distinct saturable $\mathrm{MPP}^{+}$ binding to agarose-linked NDs containing GFP-rOCT1 (Fig. 2, A and B). Binding of $\left[{ }^{3} \mathrm{H}\right] \mathrm{MPP}^{+}$to GFP-rOCT1 containing
NDs in the presence of $2 \mathrm{mM}$ nonradioactive $\mathrm{MPP}^{+}$was not statistically significantly higher compared with $\left[{ }^{3} \mathrm{H}\right] \mathrm{MPP}^{+}$ binding to filters. Per rOCT1 monomer in the NDs, $2.1 \pm 0.1$ $\mathrm{MPP}^{+}$binding sites were determined that have similar apparent $K_{\mathrm{D}}$ values that could not be discriminated. For these sites a common value of $32 \pm 3.8 \mu \mathrm{M}$ was determined (Fig. 2B; Table 1). For agarose-coupled NDs containing GFP fusion protein with the nonoligomerizing rOCT1 variant GFPrOCT1 $(6 \Delta$ C) (Keller et al., 2011), a similar common apparent $K_{\mathrm{D}}$ value and the same number of $\mathrm{MPP}^{+}$binding sites per transporter monomer were obtained (Fig. 2B; Table 1). This indicates that the determined number and affinity of $\mathrm{MPP}^{+}$ binding sites of rOCT1 reflect properties of transporter monomers. Measurements of $\mathrm{MPP}^{+}$binding to GFP-rOCT1 at $0^{\circ} \mathrm{C}$ revealed $1.2 \pm 0.1 \mathrm{MPP}^{+}$molecules per monomer and an apparent $K_{\mathrm{D}}$ value of $66 \pm 3.1 \mu \mathrm{M}$ (Fig. 2B; Table 1). This suggests that a reduced conformational flexibility of the transporter at $0^{\circ} \mathrm{C}$ prevents access to one $\mathrm{MPP}^{+}$binding site or impedes access to both identified $\mathrm{MPP}^{+}$binding sites.

Trp218 and Asp475 Are Involved in MPP ${ }^{+}$-Binding to the Same Low-Affinity MPP ${ }^{+}$Binding Site. Previously, we observed that exchange by tyrosine of Phe160 and Trp218, that were localized to outward-facing and inward-facing clefts of 3D homology models of rOCT1, increased and decreased the apparent $K_{\mathrm{m}}$ value for $\mathrm{MPP}^{+}$, respectively (Popp et al., 2005; Gorboulev et al., 2018). When Asp475, the neighboring residue of Trp218 in the 3D model of the outward-facing cleft, was replaced by glutamate, the apparent $K_{\mathrm{m}}$ for $\mathrm{MPP}^{+}$remained unchanged. In the present study we introduced the F160Y, W218Y, or D475E mutation into GFP-rOCT1 and measured $\mathrm{MPP}^{+}$binding to the mutants in NDs formed with DMPG (Fig. 2, C-F; Table 1). The mutations had no effect on incorporation of the GFP-fusion proteins into the NDs (Supplemental Table 1). As with to the apparent $K_{\mathrm{m}}$ value, the apparent $K_{\mathrm{D}}$ value for $\mathrm{MPP}^{+}$binding to the GFP-rOCT1 mutants compared with wild-type was increased in mutant F160Y, whereas it was apparently decreased in mutant W218Y (see Table 1). Noteworthy, in mutants W218Y and D475E the maximal binding $\left(B_{\max }\right)$ of about two $\mathrm{MPP}^{+}$molecule per monomer observed in wild-type rOCT1 was decreased to about one. To determine whether both mutations decrease $\mathrm{MPP}^{+}$binding at the same site, we measured $\mathrm{MPP}^{+}$binding to the double mutant W218Y/D475E. As in both single mutants, a $B_{\max }$ of about one per transporter monomer was observed, suggesting that both mutations prevent $\mathrm{MPP}^{+}$binding to the same site.

Identification of Three MPP ${ }^{+}$Binding Sites per rOCT1 Monomer in Nanodiscs Formed with 1-Palmitoyl-2oleoyl-sn-glycero-3-phosphocholine or PC, PS, and Cholesterol. Wondering why the previously described highaffinity binding site for $\mathrm{MPP}^{+}$(Gorbunov et al., 2008) was not detected after reconstitution of GFP-rOCT1 in NDs formed with DMPG, we measured $\mathrm{MPP}^{+}$binding to GFP-rOCT1 in NDs formed with DPPC, and 1-palmitoyl-2-oleoyl-sn-glycero3-phosphocholine (POPC). With these lipids, formation of NDs and incorporation of GFP-rOCT1 into NDs was similar to that with DMPG (Supplemental Fig. 3; Supplemental Table 1). In NDs formed with DPPC, a similar apparent $K_{\mathrm{D}}$ value (25.6 \pm $3.1 \mu \mathrm{M}$ vs. $31.2 \pm 3.8 \mu \mathrm{M}, n=3$ each) and a slightly higher $B_{\text {max }}$ per monomer were observed compared with NDs formed with DMPG ( $2.35 \pm 0.12$ vs. $2.08 \pm 0.07, n=3$ each, $P<0.01)$. When GFP-rOCT1 was reconstituted into NDs formed with 1-palmitoyl-2-oleoyl-sn-glycero-3-phosphocholine (POPC), 

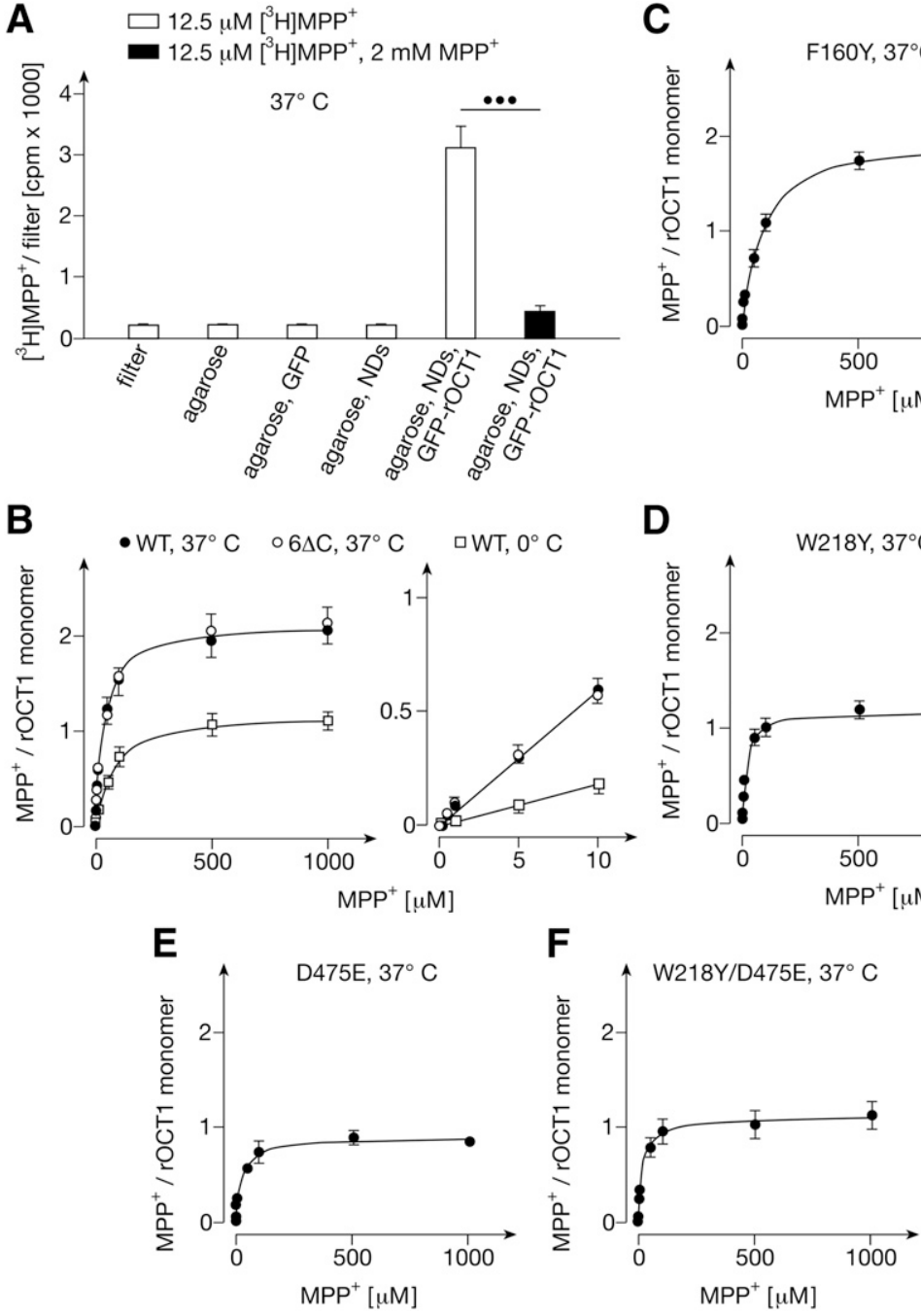
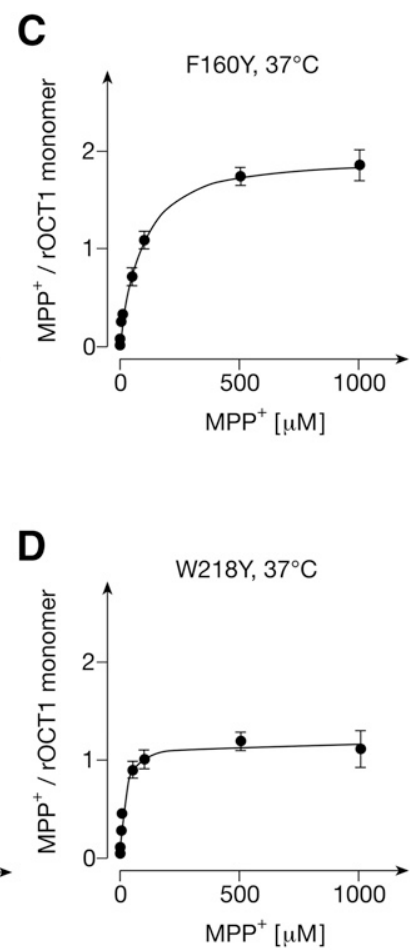

Fig. 2. Binding of $\mathrm{MPP}^{+}$to nanodiscs composed of DMPG and MSP1E3D1 containing wild-type GFP-rOCT1 and GFP-rOCT1 variants. (A) Characterization of $\mathrm{MPP}^{+}$binding to nanodics (NDs). Binding of $12.5 \mathrm{nM}\left[{ }^{3} \mathrm{H}\right] \mathrm{MPP}^{+}$in the absence and presence of $2 \mathrm{mM}$ nonradioactive $\mathrm{MPP}^{+}$was measured at $37^{\circ} \mathrm{C}$. Binding was measured to filters, $\mathrm{Ni}^{2}$ ${ }^{+}$-NTA-agarose (agarose), GFP linked to $\mathrm{Ni}^{2+}$-NTA-agarose (agarose, GFP), empty NDs linked to $\mathrm{Ni}^{2+}$-NTA-agarose (agarose, NDs), and a mixture of empty NDs and GFPrOCT1 containing NDs linked to $\mathrm{Ni}^{2+}$-NTA-agarose (agarose, NDs, GFP-rOCT1). Identical amounts of agarose beads were analyzed. Mean values \pm S.D. of four measurements are shown. $\bullet_{P}<0.001$ Student's $t$ test. (B) Effects of temperature and capability of rOCT1 to dimerize on $\mathrm{MPP}^{+}$binding. $\mathrm{MPP}^{+}$binding to GFP-rOCT1 (WT) containing NDs was measured at $37^{\circ} \mathrm{C}$ or $0^{\circ} \mathrm{C}$, and $\mathrm{MPP}^{+}$binding to nondimerizing GFP-rOCT1 $(6 \Delta \mathrm{C})$ variant was measured at $37^{\circ} \mathrm{C}$. Binding to filters was subtracted. Concentrations of rOCT1 and $\operatorname{rOCT} 1(6 \Delta \mathrm{C})$ were determined by measuring total protein in combination with densitometry of stained gels. Mean values \pm S.D. of three experiments are indicated. The curves in the left panel were obtained by fitting a "one site binding" model to the data. The straight lines in the right panel were obtained by linear regression. (C-F) Concentration dependence of $\mathrm{MPP}^{+}$binding to NDs containing mutants GFP-rOCT1(F160Y) (C), GFP-rOCT1(W218Y) (D), GFP-rOCT1(D475E) (E) or GFP-rOCT1(W218Y, D475E) (F). The measurements in (C-F) were performed and the data were calculated as in Fig. 2B. one high-affinity site $\left(B_{\max }\right.$ (high aff.) $\left.0.93 \pm 0.05\right)$ with an apparent $K_{\mathrm{D}}$ of $0.23 \pm 0.02 \mu \mathrm{M}\left(K_{\mathrm{D}}\right.$ (high aff.) $)$ could be identified per transporter monomer in addition to two low-affinity sites

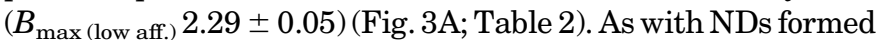
with DMPG or DPPC, the affinities of the two low-affinity sites could not be discriminated, and a common apparent $K_{\mathrm{D}}$ value $(36 \pm 2.6 \mu \mathrm{M})$ was determined. The properties of GFP-rOCT1 in NDs formed with POPC are independent of dimerization because high- and low-affinity $\mathrm{MPP}^{+}$binding sites with similar properties were obtained after reconstitution of GFP-rOCT1 and GFP-rOCT1 $(6 \Delta \mathrm{C})$ (Table 2). The data indicate that an optimal lipid environment is required for accessibility of $\mathrm{MPP}^{+}$ to the high-affinity $\mathrm{MPP}^{+}$binding site.

Next, we determined whether the nontransported inhibitor tetrabutylammonium ${ }^{+}\left(\mathrm{TBuA}^{+}\right)$(Gorboulev et al., 2018) blocks the identified $\mathrm{MPP}^{+}$binding sites. $\mathrm{TBuA}^{+}$ inhibits rOCT1-mediated $\mathrm{MPP}^{+}$uptake into proteoliposomes with an $I C_{50}$ value of $19 \mu \mathrm{M}$ (Keller et al., 2005). In NDs formed with POPC containing GFP-rOCT1, we measured the concentration dependence of $\mathrm{MPP}^{+}$binding in the presence of $2 \mathrm{mM} \mathrm{TBuA}^{+}$(Supplemental Fig. 4). In the presence of $2 \mathrm{mM} \mathrm{TBuA}^{+}, 98 \%$ of $\mathrm{MPP}^{+}$binding to the high-affinity site in the NDs and $75 \%$ of $\mathrm{MPP}^{+}$binding to the low-affinity sites was blocked.
Previously, we characterized $\mathrm{MPP}^{+}$uptake into proteoliposomes that were generated from cell-free-expressed GFP-rOCT1 and equal-weight mixtures of 1,2-diacyl-sn-glycero-3-phospho-Lserine from egg yolk (PS), 1,2-diacyl-sn-glycero-3-phosphocholine

\section{TABLE 1}

Binding of $\mathrm{MPP}^{+}$to GFP-rOCT1 wild-type and GFP-rOCT1 mutants in nanodiscs formed with DMPG

Empty NDs were formed from DMPG and His-tagged MSP1E3D1. Fusion proteins between a nondimerizing GFP mutant and rOCT1 wild-type, $\operatorname{rOCT} 1(6 \Delta \mathrm{C})$, or single point mutants of rOCT1 were reconstituted into NDs by cell-free expression in the presence of the empty NDs. The NDs were bound to $\mathrm{Ni}^{2+}$-NTA-agarose beads and precipitated. Binding of $\mathrm{MPP}^{+}$traced with $\left[{ }^{3} \mathrm{H}\right] \mathrm{MPP}^{+}$to the beads was measured at $37^{\circ} \mathrm{C}$ as described in Fig. 2B. For individual experiments, apparent $K_{\mathrm{D}}$ values and $B_{\max }$ values were determined by fitting a one-site binding model to the data. The amounts of GFP-fusion protein in the NDs were determined, and $B_{\max }$ values per transporter monomer in the preparation were calculated. Means \pm S.D. of three experiments are indicated. Significance of difference to GFP-rOCT1 was determined by one-way ANOVA with post-hoc Dunnett test $\left({ }^{* *} P<0.01\right)$.

\begin{tabular}{lll}
\hline \multicolumn{1}{c}{ GFP-Fusion Proteins } & App. $K_{\mathrm{D}}[\mu \mathrm{M}]$ & $\begin{array}{c}B_{\max }\left(\mathrm{MPP}^{+} /\right. \\
\text {Monomer) }\end{array}$ \\
\hline GFP-rOCT1 & $31.7 \pm 3.8$ & $2.08 \pm 0.07$ \\
GFP-rOCT1(6 $\triangle \mathrm{C})$ & $31.9 \pm 3.4$ & $2.10 \pm 0.05$ \\
GFP-rOCT1(F160Y) & $76.9 \pm 6.5^{* *}$ & $1.98 \pm 0.06$ \\
GFP-rOCT1(W218Y) & $15.6 \pm 1.1$ & $1.16 \pm 0.11^{* *}$ \\
GFP-rOCT1(D475E) & $24.1 \pm 3.9$ & $0.89 \pm 0.07^{* *}$ \\
GFP-rOCT1(W218Y/D475E) & $20.6 \pm 1.2$ & $1.11 \pm 0.11^{* *}$ \\
\hline
\end{tabular}



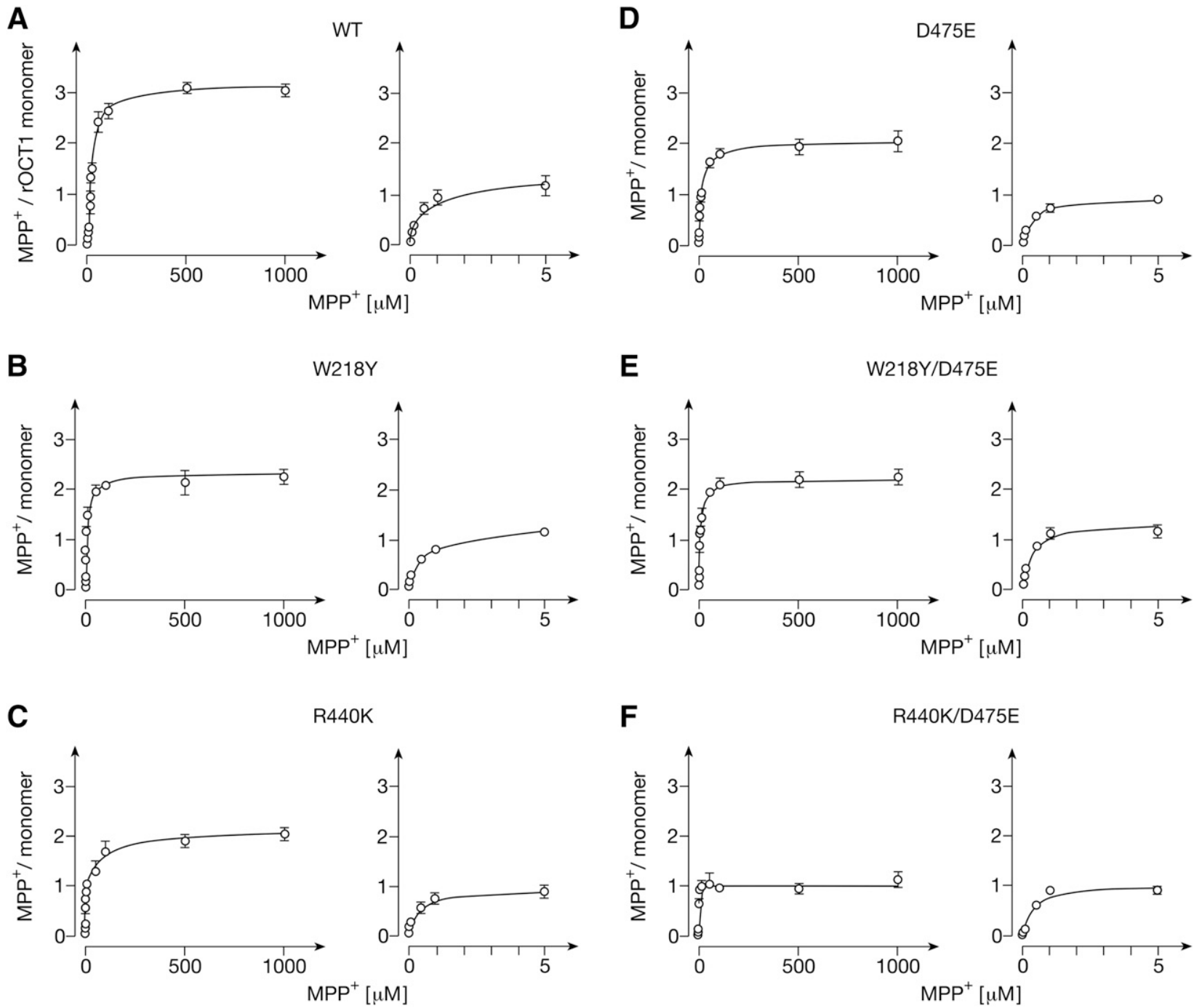

Fig. 3. Binding of $\mathrm{MPP}^{+}$to nanodiscs composed of POPC and MSP1E3D1 containing GFP-rOCT1 wild-type (A) and mutants with amino acid exchanges in positions $218(\mathrm{~B}), 440(\mathrm{C}), 475(\mathrm{D}), 218$ and $475(\mathrm{E})$, and 440 and $475(\mathrm{~F})$. His-tagged GFP-rOCT1 (WT) or GFP-rOCT1 mutants were reconstituted by cell-free expression in the presence of empty NDs formed from POPC and His-tagged MSP1E3D1. The NDs were precipitated by Ni ${ }^{2+}-\mathrm{NTA}^{2}$ agarose, and binding of $12.5 \mathrm{nM}\left[{ }^{3} \mathrm{H}\right] \mathrm{MPP}^{+}$was measured at $37^{\circ} \mathrm{C}$ in the presence of various concentrations of unlabeled $\mathrm{MPP}^{+}$. MPP ${ }^{+}$binding was measured and numbers of transporter monomers were determined as in Fig. 2B. Mean values \pm S.D. of three experiments are indicated. A two-site binding model was fitted to the data. In the right panel, $\mathrm{MPP}^{+}$binding and the fitted curve at low $\mathrm{MPP}^{+}$concentrations are shown.

from bovine brain (PC), and cholesterol (Keller et al., 2008). To evaluate whether the failure to distinguish a high-affinity $K_{\mathrm{m}}$ value for $\mathrm{MPP}^{+}$uptake in proteoliposomes could be attributable to inaccessibility of the high-affinity $\mathrm{MPP}^{+}$binding site in this lipid environment, we reconstituted GFP-rOCT1 also into NDs formed with the $\mathrm{PS} / \mathrm{PC} /$ cholesterol mixture. Under these conditions, one high-affinity $\mathrm{MPP}^{+}$binding site per transporter monomer $B_{\max }$ (high aff.) $1.01 \pm 0.19$ ) and two low-affinity $\mathrm{MPP}^{+}$binding sites per monomer ( $B_{\max }$ (low aff.) $2.06 \pm 0.19$ ) were determined as well (Fig. 4). The apparent $K_{\mathrm{D}}$ of the high-affinity binding site $(0.16 \pm 0.07$ $\mu \mathrm{M})$ was similar to NDs formed from POPC $(0.23 \pm 0.02 \mu \mathrm{M})$. At variance, for binding of $\mathrm{MPP}^{+}$to the two low-affinity sites, a lower common apparent $K_{\mathrm{D}}$ value compared with NDs formed from POPC was obtained $(9.9 \pm 2.8 \mu \mathrm{M}$ vs. $36 \pm 2.6 \mu \mathrm{M}, P<0.001$, $n=3$ each). Noteworthy, the apparent low-affinity $K_{\mathrm{D}}$ value for $\mathrm{MPP}^{+}$binding measured in NDs formed with PS/PC/cholesterol mixture was lower compared with the apparent $K_{\mathrm{m}}$ value measured in proteoliposomes formed with the same lipids (see Table $2,9.9 \pm 2.8 \mu \mathrm{M}$ vs. $19.3 \pm 3.1 \mu \mathrm{M}, P<0.001, n=3$ each).
Evidence that Arg440 Is Involved in $\mathrm{MPP}^{+}$-Binding to a Different Low-Affinity $\mathrm{MPP}^{+}$Binding Site than Trp218 and Asp475. To determine whether the mutations studied in NDs formed with DMPG exhibit effects on the highaffinity $\mathrm{MPP}^{+}$binding site, we also measured $\mathrm{MPP}^{+}$binding after reconstitution of the mutants in NDs formed with POPC (Fig. 3, B, D, and E; Fig. 5A; Table 2). In NDs formed with POPC the mutations F160Y, W218Y, D475E, and the double mutant W218Y/D475E exerted similar effects on low-affinity MPP binding as in NDs formed with DMPG. $B_{\max }$ of lowaffinity $\mathrm{MPP}^{+}$binding per monomer was reduced from about two to one in mutants W218Y, D475E, and W218Y/D475E, and the affinity for low-affinity binding was decreased in mutant F160Y. $B_{\max }$ for high-affinity binding remained unaltered. The apparent $K_{\mathrm{D}}$ for high-affinity binding of $\mathrm{MPP}^{+}$ was increased in mutant W218Y, suggesting an allosteric effect of this mutation on the high-affinity $\mathrm{MPP}^{+}$binding site.

Trying to localize the second low-affinity and the highaffinity $\mathrm{MPP}^{+}$binding site, we also analyzed mutations of 
TABLE 2

Comparison of $\mathrm{MPP}^{+}$binding to GFP-rOCT1 wild-type and mutants in NDs with $\mathrm{MPP}^{+}$uptake into proteoliposomes

NDs were prepared with POPC. The MPP ${ }^{+}$binding measurements are shown in Figs. 3 and 5. $B_{\max (\text { high aff.) }}$ and $B_{\max \text { (low aff.) }}$ per rOCT1 monomer are indicated. Purified GFPrOCT1 wild-type and mutants were reconstituted into proteoliposomes formed with cholesterol, PC, and PS. The MPP ${ }^{+}$uptake measurements are presented in Figs. 6 and 8. Means \pm S.D. of three experiments are shown.

\begin{tabular}{|c|c|c|c|c|c|c|}
\hline Fusion Proteins & App. $K_{\mathrm{D} 1 \text { (High. Aff.) }}$ & App. $K_{\mathrm{D} 2}$ (Low Aff.) & $\begin{array}{c}B_{\max } \text { (High Aff.) } \mathrm{MPP}^{+} \\
\text {per Monomer }\end{array}$ & $\begin{array}{l}B_{\max } \text { (Low Aff.) MPP } \mathrm{MP}^{+} \\
\text {per Monomer }\end{array}$ & App. $K_{\mathrm{m}}$ of $\mathrm{MPP}^{+}$Uptake & $\begin{array}{l}\text { Min. Turnover } \\
\text { of Mpp }{ }^{+} \text {Uptake } \\
\text { per Second and } \\
\text { Monomer }\end{array}$ \\
\hline & $\mu M$ & $\mu M$ & & & $\mu M$ & \\
\hline GFP-rOCT1 & $0.23 \pm 0.02$ & $36 \pm 2.6$ & $0.93 \pm 0.05$ & $2.29 \pm 0.05$ & $19 \pm 3.1$ & $17 \pm 2.1$ \\
\hline GFP-rOCT1 $(6 \Delta \mathrm{C})$ & $0.25 \pm 0.01$ & $39 \pm 5.8$ & $1.04 \pm 0.04$ & $2.10 \pm 0.04$ & & \\
\hline GFP-rOCT1(F160Y) & $0.28 \pm 0.02$ & $86 \pm 5.6^{* *}$ & $1.07 \pm 0.04$ & $2.04 \pm 0.05^{*}$ & $60 \pm 4.6^{* *}$ & $20 \pm 3.0$ \\
\hline GFP-rOCT1(W218Y) & $0.34 \pm 0.05^{*}$ & $21 \pm 3.0$ & $0.97 \pm 0.18$ & $1.36 \pm 0.17^{* *}$ & $15 \pm 1.0$ & $9.6 \pm 1.3^{* *}$ \\
\hline GFP-rOCT1(D475E) & $0.21 \pm 0.04$ & $28 \pm 4.7$ & $0.81 \pm 0.06$ & $1.29 \pm 0.06^{* *}$ & $18 \pm 1.2$ & $3.5 \pm 0.25^{* *}$ \\
\hline GFP-rOCT1(W218Y/D475E) & $0.20 \pm 0.03$ & $21 \pm 2.9$ & $1.15 \pm 0.13$ & $1.05 \pm 0.12^{* *}$ & $20 \pm 3.4$ & $3.7 \pm 0.38^{* *}$ \\
\hline GFP-rOCT1(R440K) & $0.30 \pm 0.06$ & $81 \pm 15^{* *}$ & $0.89 \pm 0.06$ & $1.24 \pm 0.06^{* *}$ & $69 \pm 4.5^{* *}$ & $12 \pm 1.6^{* *}$ \\
\hline GFP-rOCT1(R440K/D475E) & $0.32 \pm 0.06$ & Not detectable & $1.10 \pm 0.05$ & Not detectable & $26 \pm 4.7$ & $0.73 \pm 0.15^{* *}$ \\
\hline GFP-rOCT1(L447Y) & $0.22 \pm 0.06$ & $8.2 \pm 2.0 * *$ & $0.81 \pm 0.20$ & $2.08 \pm 0.20$ & $8.6 \pm 0.3^{* *}$ & $18 \pm 0.41$ \\
\hline GFP-rOCT1(L447F) & $0.26 \pm 0.02$ & $14 \pm 1.6^{* *}$ & $0.94 \pm .21$ & $2.00 \pm 0.08^{*}$ & $8.7 \pm 0.4^{* *}$ & $16 \pm 1.0$ \\
\hline GFP-rOCT1(Q448E) & $0.14 \pm 0.01$ & $19 \pm 2.3^{*}$ & $0.92 \pm 0.19$ & $2.19 \pm 0.18$ & $13 \pm 0.9$ & $19 \pm 1.9$ \\
\hline
\end{tabular}

$* P<0.05 ; * * P<0.01$ Significance of difference to GFP-rOCT1 determined by one-way ANOVA with post-hoc Dunnett test.

Arg440, Leu447, and Gln448 which also have been localized within the outward-open cleft of our rOCT1 model (Volk et al., 2009; Gorboulev et al., 2018) (Fig. 3C; Fig. 5, B-D; Table 2). In mutants L447Y, L447F, and Q448E, maximal binding to the low- and high-affinity binding sites per monomer was not altered; however, the apparent $K_{\mathrm{D}}$ values for low-affinity binding were decreased. The data indicate that Leu447 and Gln448 are not essential for low- or high-affinity binding, but that mutations in these positions may exhibit allosteric effects on $\mathrm{MPP}^{+}$binding sites. After replacement of Arg440 by lysine, $B_{\max }$ of low-affinity binding was decreased from $2.29 \pm 0.05$ to $1.24 \pm 0.06$ per monomer (Fig. 3C; Table 2). The common apparent $K_{\mathrm{D}}$ value for low-affinity binding of $\mathrm{MPP}^{+}$was doubled, whereas high-affinity binding remained unchanged. To determine whether the R440K mutation blunted $\mathrm{MPP}^{+}$ binding to a low-affinity site different from the $\mathrm{D} 475 \mathrm{E}$ mutation, we measured $\mathrm{MPP}^{+}$binding to double mutant R440K/D475E (Fig. 3F; Table 2). Noteworthy, in this mutant

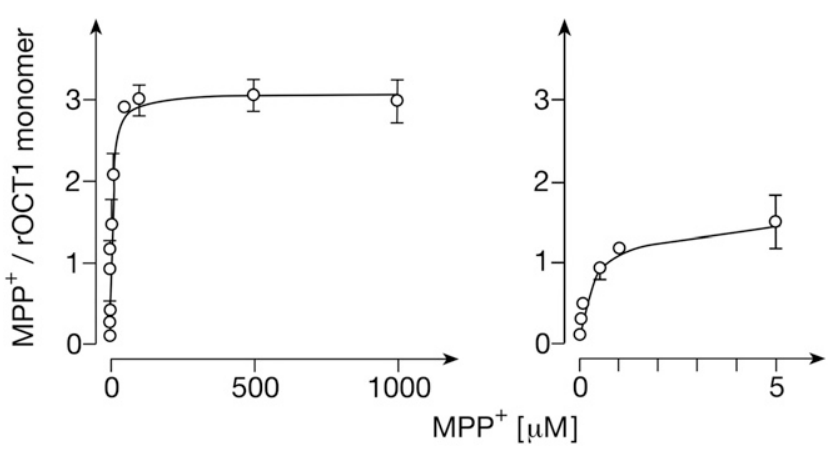

Fig. 4. Binding of $\mathrm{MPP}^{+}$to GFP-rOCT1 in nanodiscs formed with PC, PS, and cholesterol and MSP1E3D1. His-tagged GFP-rOCT1 was reconstituted by cell-free expression in the presence of empty NDs formed from PC, PS, and cholesterol and His-tagged MSP1E3D1. The NDs were precipitated by $\mathrm{Ni}^{2+}$-NTA agarose beads and binding of $12.5 \mathrm{nM}\left[{ }^{3} \mathrm{H}\right] \mathrm{MPP}^{+}$ was measured at $37^{\circ} \mathrm{C}$ in the presence of various concentrations of unlabeled $\mathrm{MPP}^{+}$. $\mathrm{MPP}^{+}$binding was measured and numbers of transporter monomers were determined as in Fig. 2B. Mean values \pm S.D. of three experiments are indicated. A two-site binding model was fitted to the data. In the right panel, $\mathrm{MPP}^{+}$binding and the fitted curve at low MPP ${ }^{+}$ concentrations are shown. no low-affinity $\mathrm{MPP}^{+}$binding was detectable, whereas $B_{\max }$ per transporter monomer of high-affinity $\mathrm{MPP}^{+}$binding was not altered.

Evidence That Both Low-Affinity MPP $^{+}$Binding Sites Are Involved in Transport. To elucidate the relevance of the $\mathrm{MPP}^{+}$binding sites for transport, we reconstituted cell-free-expressed GFP-rOCT1 wild-type and GFP-rOCT1 mutants into proteoliposomes and measured the substrate dependence of $\mathrm{MPP}^{+}$uptake. The proteoliposomes were formed from PS, PC, and cholesterol, and $\mathrm{MPP}^{+}$ uptake was measured after a 1 -second incubation at $37^{\circ} \mathrm{C}$ in the presence of an inside-negative potassium diffusion potential, as described for rOCT1 (Keller et al., 2008). As with rOCT1 in proteoliposomes (Keller et al., 2005, 2008) and rOCT1 expressed in oocytes or human embryonic kidney (HEK) 293 cells (Busch et al., 1996; Gorboulev et al., 2018), for GFP-rOCT1 in proteoliposomes, a hyperbolic concentration dependence was observed that could be fitted to the Michaelis-Menten equation (Fig. 6A). No indication of an additional high-affinity uptake was detectable. For GFPrOCT1, an apparent $K_{\mathrm{m}}$ value of $19 \pm 3.1 \mu \mathrm{M}(n=3)$ was obtained that was lower than the apparent $K_{\mathrm{m}}$ of $35 \pm 1.5 \mu \mathrm{M}$ determined for cell-free-expressed rOCT1 in proteoliposomes (Keller et al., 2008).

In mutants $\mathrm{W} 218 \mathrm{Y}$ and $\mathrm{R} 440 \mathrm{~K}$, where binding of $\mathrm{MPP}^{+}$to one or the other low-affinity binding site was largely impaired, and which led to $41 \%$ (W218Y) and $46 \%$ (R440K) decrease in total low-affinity binding, the $V_{\max }$ of $\mathrm{MPP}^{+}$transport was reduced by $44 \%(\mathrm{~W} 218 \mathrm{Y}$ ) and $29 \%(\mathrm{R} 440 \mathrm{~K})$ (Fig. 6 , B and C; Fig. 7A; Table 2). When the Trp218- and Asp475-related binding site was blocked by exchange of Asp475 with glutamate, total low-affinity binding was decreased by $44 \%$, similar to the W218Y mutation; however, a more pronounced $79 \%$ decrease of $V_{\max }$ was observed (Fig. 6E; Fig. 7A; Table 2). The more pronounced effect on $V_{\max }$ reflects the dual role of Asp475 for substrate affinity and transport-related conformational changes (Egenberger et al., 2012). The double mutant W218Y/D475E exhibited properties similar to the D475E mutant, showing a $54 \%$ reduction in low-affinity binding and a $78 \%$ reduction in $V_{\max }$ (Fig. 6D; Fig. 7A; Table 2). In double mutant R440K/D475E, for which no low-affinity 
A
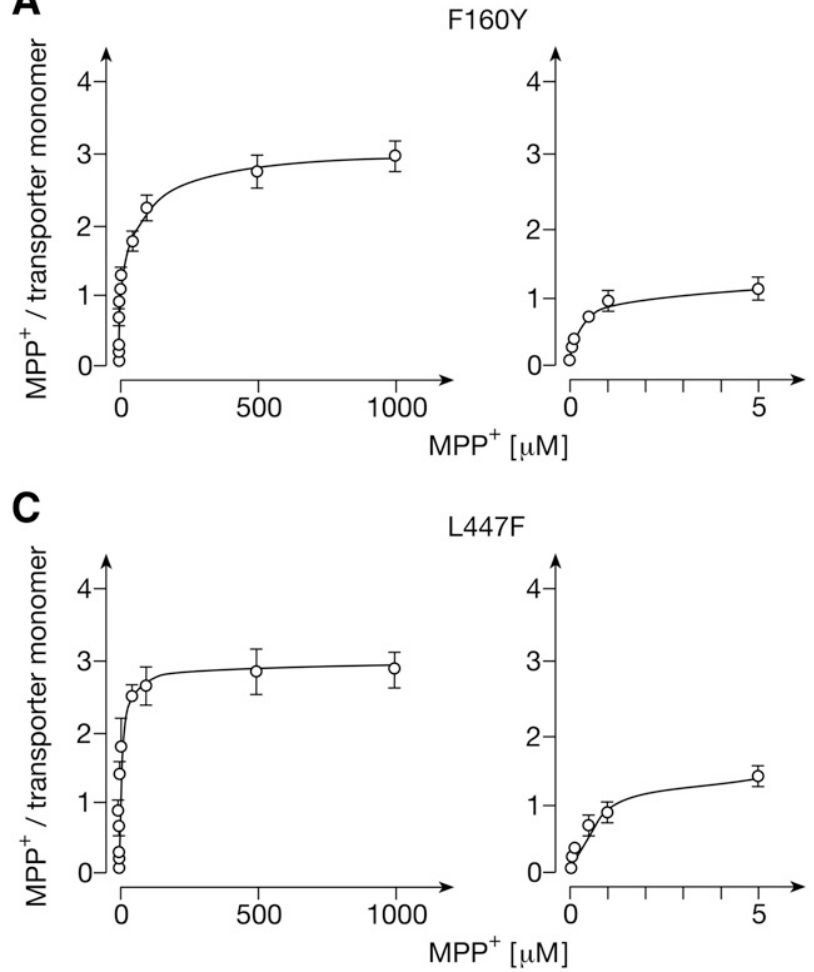

B
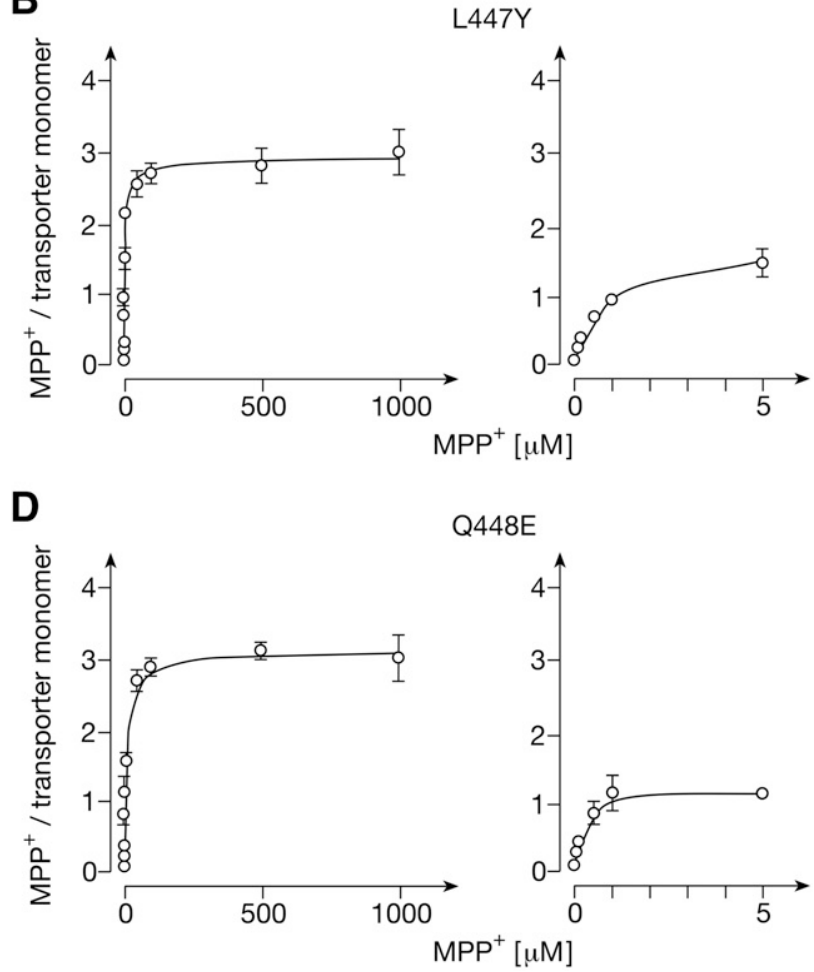

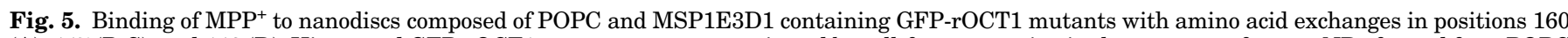

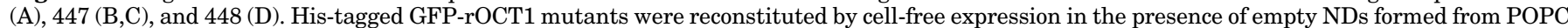

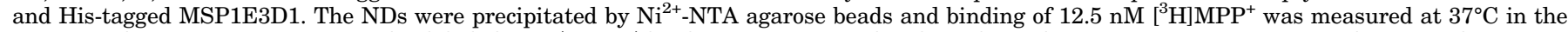

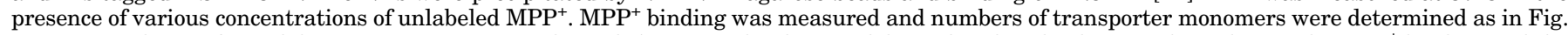

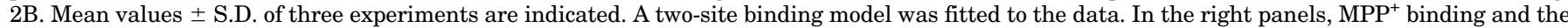
fitted curves at low $\mathrm{MPP}^{+}$concentrations are shown.

binding was detected, the $V_{\max }$ was reduced by $96 \%$ (Fig. $6 \mathrm{~F}$; Fig. 7A; Table 2). The data indicate that $\mathrm{MPP}^{+}$binding to the two identified low-affinity sites is critically involved in translocation.

In mutants W218Y, D475E, and W218Y/D475E, the apparent $K_{\mathrm{m}}$ value for $\mathrm{MPP}^{+}$uptake was not altered significantly, whereas in mutant $\mathrm{R} 440 \mathrm{~K}$, the apparent $K_{\mathrm{m}}$ value was increased 3-fold (Fig. 7B). The effects on apparent $K_{\mathrm{m}}$ have some similarity to the effects on the common apparent $K_{\mathrm{D}}$ (low aff.) value, which was not altered in mutant $\mathrm{D} 475 \mathrm{E}$, decreased by $42 \%$ in mutants W218Y and W218Y/D475E, and 2.3-fold increased in mutant R440K. The small differences between the effects of the R440K and W218Y/D475E mutations on apparent

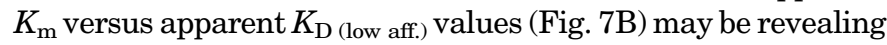
the difference in lipid composition between the NDs and proteoliposomes and/or that the affinity for substrate binding may not exclusively determine the $K_{\mathrm{m}}$ for translocation.

In mutants F160Y, L447Y, L447F, and Q448E no significant changes in the determined total number of three binding sites per transporter monomer and the $V_{\max }$ values for $\mathrm{MPP}^{+}$ transport were observed (Figs. 5 and 8; Table 2). However, apparent $K_{\mathrm{D} \text { (low aff.) }}$ and apparent $K_{\mathrm{m}}$ values were increased in mutant F160Y and decreased in mutants L447Y and L447F. The data support the interpretation that the investigated mutations of Phe160 and Leu447 induce allosteric effects on $\mathrm{MPP}^{+}$binding to the low-affinity sites that are critically involved in translocation.

Docking of $\mathrm{MPP}^{+}$to Modeled Outward-Open and Inward-Open Clefts of rOCT1. Previously, we generated a
3D homology model of the inward-open conformation of rOCT1 on the basis of the crystal structure of lactose permease from $E$. coli that belongs to the MFS superfamily like the OCTs (Popp et al., 2005). In addition, we also built a 3D model of rOCT1 in the outward-open conformation by assuming a rigidbody movement of the six N-terminal helices of rOCT1 with respect to the six $\mathrm{C}$-terminal helices, a model similar to that generated modeled and experimentally verified for lactose permease (Holyoake and Sansom, 2007; Kaback et al., 2007; Volk et al., 2009). Using as templates the crystal structures of various transporters of the MFS superfamily, an outwardopen 3D model of human OCT1 (hOCT1) was built (Dakal et al., 2017). In this hOCT1 model, the locations of Phe160, Trp218, Arg440, and Asp475 within the outward-open cleft of rOCT1 were confirmed (Gorboulev et al., 2018). In the outward-open models of rOCT1 and hOCT1 Trp218 (Trp217 in hOCT1) and Asp475 (Asp474) were in close proximity, whereas Arg440 (Arg439) was located more distantly to the two other residues. This is consistent with our mutagenesis data suggesting that Asp475 and Trp218 contribute to the same low-affinity $\mathrm{MPP}^{+}$binding site, whereas Arg440 is important for integrity of the second low-affinity $\mathrm{MPP}^{+}$ binding site. In the inward-open cleft model of rOCT1, Asp475 is located distantly from Trp218 and Arg440 (Volk et al., 2009). This suggests that in the reconstituted NDs, $\mathrm{MPP}^{+}$binding to the outward-open conformation has been analyzed. Trying to obtain additional insight on $\mathrm{MPP}^{+}$ binding close to Trp218, Arg440 and Asp475, we performed in silico docking simulations of $\mathrm{MPP}^{+}$into the outward-facing 

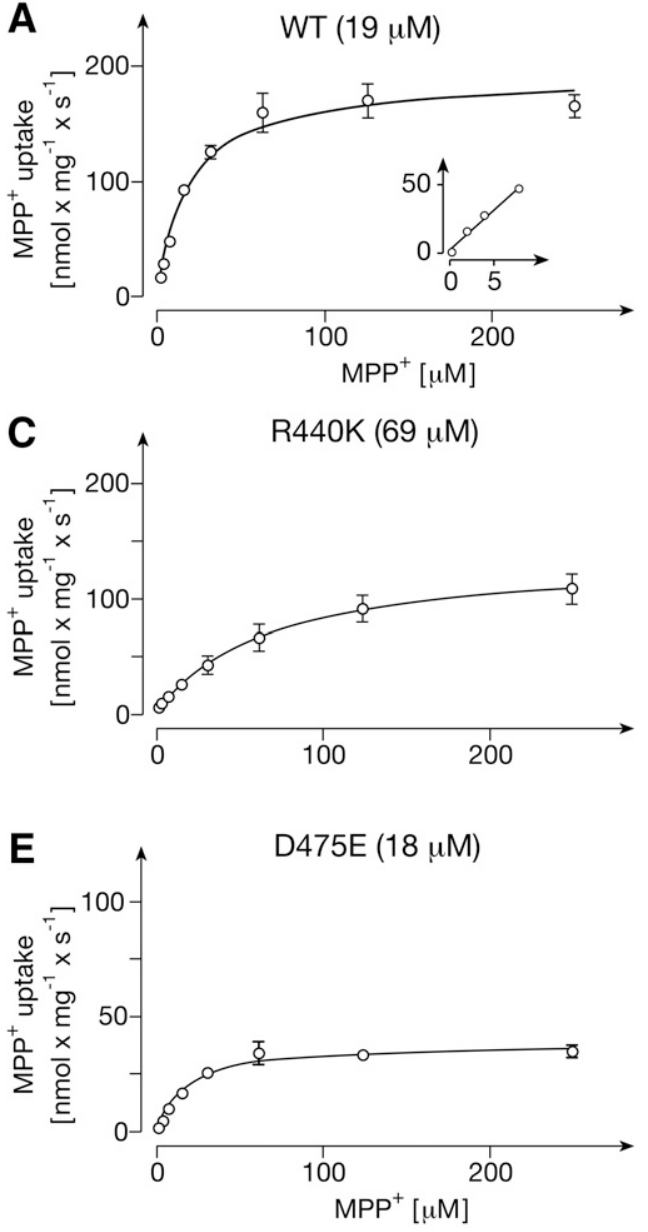
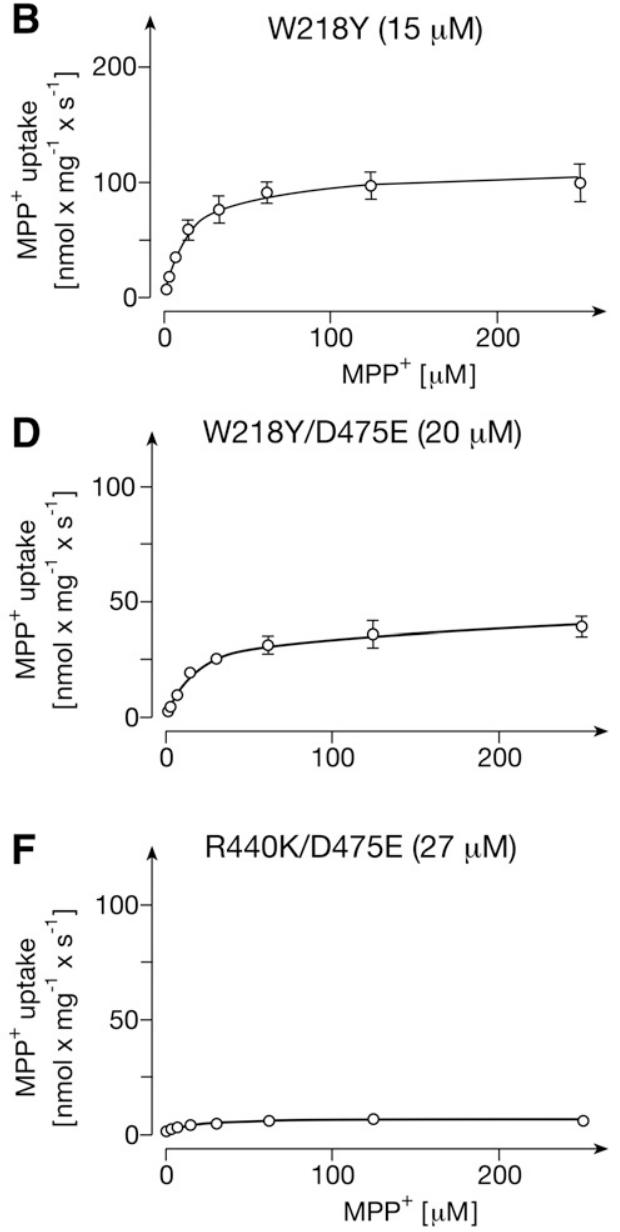

Fig. 6. Transport of $\mathrm{MPP}^{+}$into proteoliposomes containing GFP-rOCT1 wildtype (A) or mutants with amino acid exchanges in positions 218 (B), 440 (C), 475 (E), 218 and 475 (D), and 440 and 475 (F). His-tagged GFP-rOCT1 (WT) or GFP-rOCT1 mutants were reconstituted into proteoliposomes formed with cholesterol, PC, and PS. Uptake of 12.5 $\mathrm{nM}\left[{ }^{3} \mathrm{H}\right] \mathrm{MPP}^{+}$into proteoliposomes was measured after a 1-second incubation at $37^{\circ} \mathrm{C}$ in the presence of an outwarddirected $\mathrm{K}^{+}$-diffusion potential and various concentrations of unlabeled $\mathrm{MPP}^{+}$. Nonspecific uptake measured in the presence of quinine was subtracted. Mean values \pm S.D. of three experiments are indicated. The MichaelisMenten equation was fitted to the data. The apparent $K_{\mathrm{m}}$ values are indicated in parenthesis. and inward-facing clefts of our 3D homology models of rOCT1. To screen potential binding sites close to the above listed residues, docking was restrained to within a $7-\AA$ radius around these residues. The docking simulations have been deposited at the Protein Model Database (see end of paragraph).* The 30 docked $\mathrm{MPP}^{+}$molecules with the highest docking energies in both conformations were analyzed (Fig. 9). In the outward-open and inward-open rOCT1 conformations, 26 and 22 molecules were found docked within the cleft, respectively. In the outward-open cleft $18 \mathrm{MPP}^{+}$molecules were located close to Trp218 and Asp475, whereas in the inward-open conformation $10 \mathrm{MPP}^{+}$were close to both Trp218 and Asp475. Hence, the docking experiments did not allow us to decide whether low-affinity binding of two $\mathrm{MPP}^{+}$ molecules to the outward-open or inward-open cleft was determined by the binding measurements of GFP-rOCT1 in NDs. [*Protein Model database (https://bioinformatics.cineca. it/PMDB/main.php): accession no. PM0081570, $\mathrm{MPP}^{+}$docking to the outward-open conformation of rOCT1; accession no. PM0081569, $\mathrm{MPP}^{+}$docking to inward-open conformation of rOCT1.]

\section{Discussion}

On the basis of mutagenesis experiments in rOCT1 combined with binding and transport measurements of the model cation $\mathrm{MPP}^{+}$, our study provides novel insight into recognition and transport of drugs by polyspecific organic cation transporters (OCTs) of the SLC22 transporter family. The experiments were performed with cell-free-expressed GFP-rOCT1 fusion protein that was reconstituted into NDs and proteoliposomes. Evidence is provided that each rOCT1 monomer contains two low-affinity $\mathrm{MPP}^{+}$binding sites that are involved in $\mathrm{MPP}^{+}$transport and one high-affinity $\mathrm{MPP}^{+}$binding site that does not directly participate in transport but may mediate allosteric effects on the low-affinity $\mathrm{MPP}^{+}$binding sites. It cannot be excluded that the determined numbers of $\mathrm{MPP}^{+}$binding sites per transporter monomer are underestimated, because it has not been proven experimentally that all transporter monomers in the NDs are functional. However, functionality of all transporters in our preparation is probable, because the reconstitution of GFP-rOCT1 into NDs was performed by cotranslational insertion during cellfree expression of the transporter in the absence of detergent (Roos et al., 2013). Thus, post-translational protein modifications and protein aggregation causing protein heterogeneities were avoided. More importantly, because only about $1 \%$ of the NDs contained GFP-rOCT1, a homogeneous population of NDs containing functional transporter is expected.

The identified high-affinity $\mathrm{MPP}^{+}$binding site was only accessible in specific lipid environments, namely in NDs formed with POPC or a mixture of cholesterol, PC, and PS, but not with DMPG or DPPC. The lipid dependence suggests that a minimal membrane fluidity (POPC vs. DPPC) and/or an 

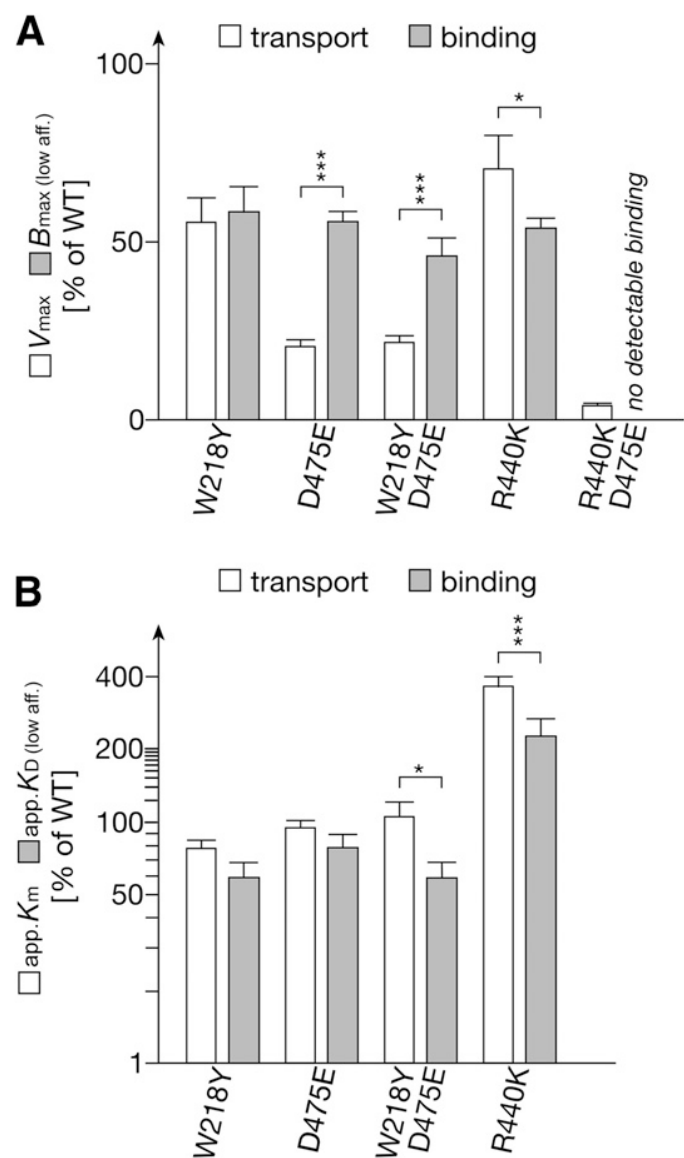

Fig. 7. Comparison between kinetic constants of GFP-rOCT1 wild-type and mutants with amino acid exchanges in positions 218,440 , and 475 determined for $\mathrm{MPP}^{+}$uptake into proteoliposomes and for $\mathrm{MPP}^{+}$ binding to NDs formed with POPC. (A) Comparison between $V_{\max }$ values for $\mathrm{MPP}^{+}$uptake and $B_{\max }$ values for $\mathrm{MPP}^{+}$binding. (B) Comparison between apparent $K_{\mathrm{m}}$ values for $\mathrm{MPP}^{+}$uptake and apparent $K_{\mathrm{D}}$ values for low-affinity $\mathrm{MPP}^{+}$binding. The compiled data are presented in Figs. 3 and 6 . The compared mean \pm S.D. values were calculated from three individual experiments. The values are shown in Table 2 . $\left({ }^{*} P<0.05\right.$; $* * * P<0.001$; significance of differences between $V_{\max }$ and $B_{\max }$ values and between apparent $K_{\mathrm{m}}$ and apparent $K_{\mathrm{D}}$ (low-aff.) values of individual mutants that were determined by two-way ANOVA using Bonferroni correction.)

optimal membrane thickness (DMPG vs. POPC) is required for an in vivo-like transporter conformation with an accessible high-affinity binding site. Phospholipid binding to a lipid binding site of rOCT1 as has been described for some transporters (Laganowsky et al., 2014), is also possible.

The involvement of individual low-affinity sites in transport can be concluded from the observed parallel decrease in $B_{\max }$ of $\mathrm{MPP}^{+}$binding and $V_{\text {max }}$ of $\mathrm{MPP}^{+}$transport after inactivation of one or the other low-affinity $\mathrm{MPP}^{+}$binding site in mutants W218Y and R440K, as well as the total absence of uptake after inactivation of both low-affinity binding sites in the double mutant R440K/D475E. A direct involvement of the high-affinity $\mathrm{MPP}^{+}$binding site in transport could be excluded because $B_{\max }$ of high-affinity $\mathrm{MPP}^{+}$binding remained unchanged when both low-affinity $\mathrm{MPP}^{+}$binding sites were blocked and $\mathrm{MPP}^{+}$transport was abolished. In addition, neither a high-affinity $\mathrm{MPP}^{+}$transport site nor positive cooperativity could be detected by measuring the substrate dependence of $\mathrm{MPP}^{+}$uptake in proteoliposomes (Fig. 6A) or in those rOCT1-expressing oocytes in which high-affinity $\mathrm{MPP}^{+}$binding sites have been identified (Busch et al., 1996; Gorbunov et al., 2008).

The properties of rOCT1 we discovered provide a molecular framework for understanding how various factors can influence the affinities of inhibitors. For example, for inhibition of $\mathrm{MPP}^{+}$uptake into rOCT1-expressing HEK293 cells by a nontransported inhibitor, widely divergent $\mathrm{IC}_{50}$ values were obtained when different concentrations of $\mathrm{MPP}^{+}$far below the apparent $K_{\mathrm{m}}$ for $\mathrm{MPP}^{+}$uptake were used for uptake measurements (Gorboulev et al., 2018). Under these conditions only one low-affinity $\mathrm{MPP}^{+}$binding site is supposed to be involved in transport and a different occupation is assumed for the high-affinity $\mathrm{MPP}^{+}$binding site. A high complexity of drugdrug interaction should then be anticipated, considering that OCTs contain both allosteric high-affinity cation binding sites (Gorbunov et al., 2008; Minuesa et al., 2009) as well as partially overlapping, transport-related, low-affinity cation binding sites, and that cationic drugs with largely different structures may interact with the high- and low-affinity sites. (Koepsell, 2013). Hence, the procedures recommended by the U.S. Food and Drug Administration (US Food and Drug Administration, 2012, 2017) and European Medicine Agencies and in the literature to determine whether NMEs interact with hOCT1 or hOCT2 by measuring inhibition of uptake of one concentration of $\mathrm{MPP}^{+}$or metformin as test substrate are insufficient for drug development, because effects of substrate structure and functionally relevant high-affinity inhibition are missed (Koepsell, 2018). Considering the complexity of drug interactions with OCTs, more sophisticated approaches are required. Hence, we suggest employing three structurally diverse substrates including a clinically relevant compound at three different concentrations far below the respective $K_{\mathrm{m}}$ values for in vitro testing of NMEs by inhibition experiments. Pharmacophore models based on the different experimental conditions may be generated and applied one after the other to achieve a predictive potential that is high enough to justify in silico exclusion of NMEs from in vitro testing. Such a line of action is mandatory for in silico prediction, because pharmacophore models so far described for hOCT1 and hOCT2 are insufficient, because they are based on uptake measurements performed at a single substrate concentration that is close to the respective $K_{\mathrm{m}}$ value. By these models only $70 \%-82 \%$ of the interacting drugs have been identified (Koepsell, 2018).

The current understanding of the molecular mechanism by which human polyspecific drug transporters recognize compounds with different molecular structures has been limited by its reliance on modeling and on indirect experimental evidence. So far, crystal structures of mammalian polyspecific transporters and of transporter-ligand complexes have been missing, and homology models derived from the crystal structures of bacterial transporters belonging to the same superfamily and docking experiments of ligands to homology models have provided only limited information. Extensive mutagenesis studies have been performed only with a few polyspecific drug transporters to measure the effects of mutations on apparent $K_{\mathrm{m}}$ values of substrates and on $\mathrm{IC}_{50}$ values for inhibition of transport. These measurements depicted secondary effects of the mutations rather than direct effects on substrate- or inhibitor binding. Recently, crystal structures of four dipeptide complexes with different conformations of the proton-dependent peptide transporter Pep $\mathrm{T}_{\mathrm{StT}}$ from Streptococcus thermophiles were reported (Martinez 

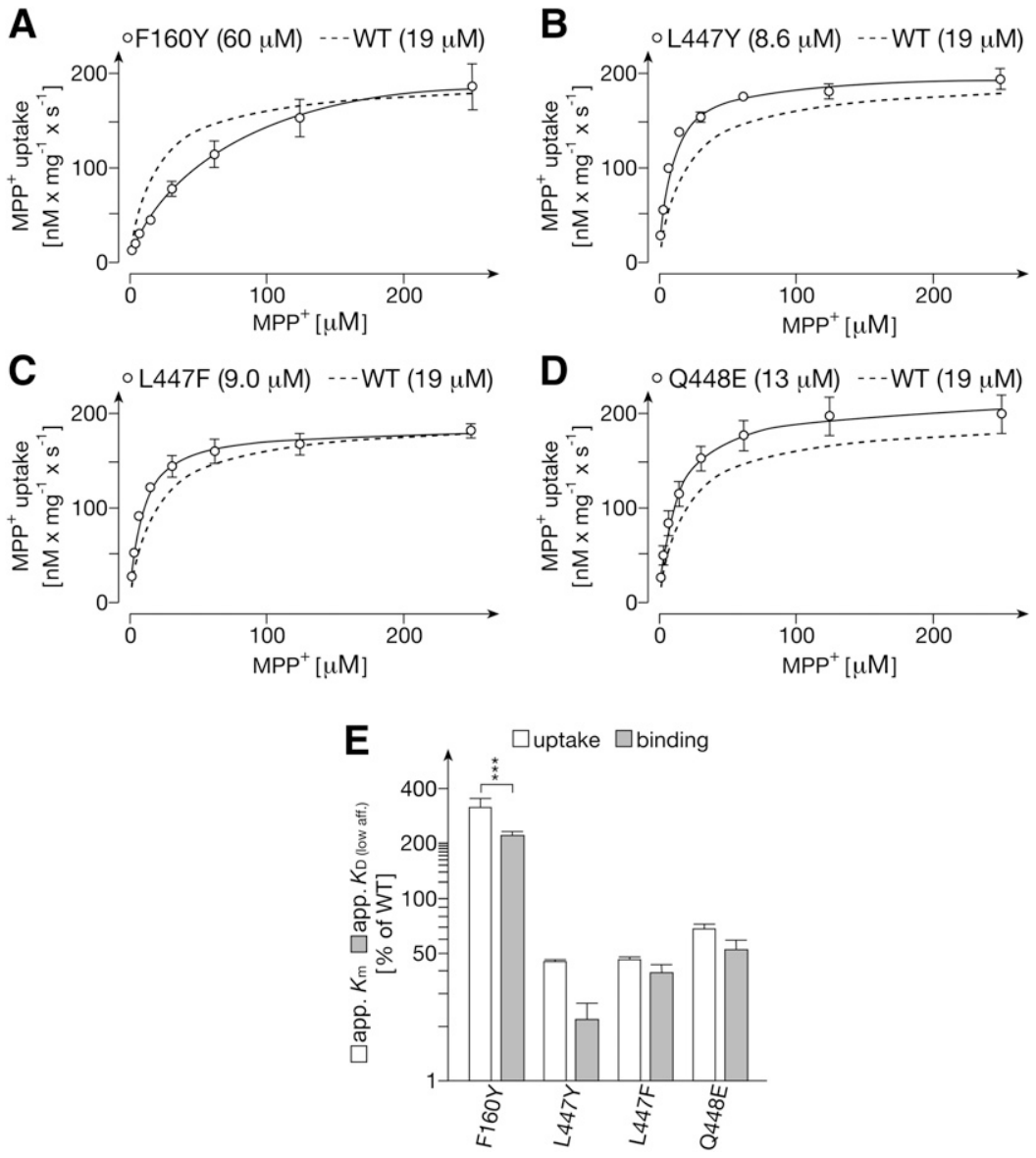

Molledo et al., 2018). The data suggest that binding of different dipeptides to $\mathrm{Pep}_{\mathrm{ST}}$ occurs at the same binding site and is enabled by movements of the interacting amino acid residues in combination with adjustments of dipeptide positions and interposition of water molecules. Remarkably, the $\mathrm{EC}_{50}$ values obtained for the interaction of the cocrystallized dipeptides exhibiting different but not completely divergent structures from solubilized $\mathrm{Pep}_{\mathrm{StT}}$ ranged between 0.56 and $>50 \mathrm{mM}$. In contrast, organic cations with completely different molecular structures, such as $\mathrm{TBuA}^{+}$, metformin ${ }^{+}$, and $\mathrm{MPP}^{+}$, interacting with OCTs had $\mathrm{EC}_{50}$ values that ranged between subnanomolar and millimolar concentrations. Consistent with this different degree of polyspecificity between proton-peptide cotransporters and OCTs, we have provided evidence for the existence of different cation binding sites in rOCT1. Each of these binding sites in rOCT1-and presumably different binding sites in all OCTs-may recognize a group of structurally related compounds employing mechanisms as described for PepT $\mathrm{T}_{\mathrm{StT}}$. In total this allows the recognition of a large variety of structurally diverse compounds by OCTs.

By measuring binding of $\mathrm{MPP}^{+}$to GFP-rOCT1 in NDs, binding to low-affinity binding sites within either the outward-open or the inward-open cleft of the transporter can be determined. On the basis of homology modeling and docking data, no distinction between binding to the outwardopen and inward-open cleft can be made. Likewise, the determined apparent $K_{\mathrm{m}}$ values for $\mathrm{MPP}^{+}$uptake and the apparent $K_{\mathrm{D}}$ values for low-affinity $\mathrm{MPP}^{+}$binding do not allow
Fig. 8. Transport of $\mathrm{MPP}^{+}$into proteoliposomes containing GFP-rOCT1 mutants with exchanges of amino acids in positions 160, 447, and 448 (A-D) and comparison between apparent $K_{\mathrm{m}}$ values for uptake and apparent $K_{\mathrm{D}}$ (low aff.) values for binding (E). GFP-rOCT1 mutants were reconstituted into proteoliposomes formed with PC, PS, and cholesterol. Uptake of $12.5 \mathrm{nM}\left[{ }^{3} \mathrm{H}\right] \mathrm{MPP}^{+}$into proteoliposomes was measured after a 1 -second incubation at $37^{\circ} \mathrm{C}$ in the presence of an outward-directed $\mathrm{K}^{+}$-diffusion potential and various concentrations of unlabeled $\mathrm{MPP}^{+}$. (A-D) The Michaelis-Menten equation was fitted to the compiled data sets. The curve obtained for the concentration dependence of $\mathrm{MPP}^{+}$uptake in proteoliposomes containing GFP-rOCT1 (WT) is indicated for comparison. The apparent $K_{\mathrm{m}}$ values (see Table 2) are indicated in parenthesis. (E) Comparison of apparent $K_{\mathrm{m}}$ values for $\mathrm{MPP}^{+}$uptake into proteoliposomes with apparent $K_{\mathrm{D}}$ values for low affinity $\mathrm{MPP}^{+}$binding to NDs formed with POPC. Mean values \pm S.D. from three experiments are indicated. Significance of difference between apparent $K_{\mathrm{m}}$ and apparent $K_{\mathrm{D}}$ (low aff.) values of individual mutants were determined by two-way ANOVA with Bonferroni correction $(* * * P<0.001)$. For mutant GFPrOCT1(L447Y) significance of a 2-fold difference between

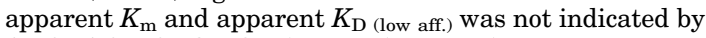
ANOVA but by Student's $t$ test $(P<0.01)$. a distinction between binding to the outward-open or inwardopen cleft because we do not know whether rOCT1-mediated cellular influx and efflux of $\mathrm{MPP}^{+}$are asymmetric and have different $K_{\mathrm{m}}$ values for $\mathrm{MPP}^{+}$transport and/or different $K_{\mathrm{D}}$ values for low-affinity $\mathrm{MPP}^{+}$binding. The observation that apparent $K_{\mathrm{m}}$ values of 35 and $19 \mu \mathrm{M}$ for $\mathrm{MPP}^{+}$uptake in proteoliposomes containing cell-free expressed rOCT1 (Keller et al., 2008) or GFP-rOCT1 were higher than the apparent $K_{\mathrm{m}}$ values measured for rOCT1-mediated $\mathrm{MPP}^{+}$uptake in oocytes $(4.9 \mu \mathrm{M})$ and HEK293 cells $(3.9 \mu \mathrm{M})$ could indicate that rOCT1 in the proteoliposomes is oriented inside-out and the $K_{\mathrm{m}}$ for $\mathrm{MPP}^{+}$efflux is higher compared with influx. However, different properties of rOCT1 in proteoliposomes compared with rOCT1 in native plasma membranes cannot be excluded. Because a high-affinity $\mathrm{MPP}^{+}$binding site on rOCT1 expressed in oocytes was identified after short-term extracellular application of $\mathrm{MPP}^{+}$(Gorbunov et al., 2008), it is highly probable that the detected high-affinity $\mathrm{MPP}^{+}$binding site is accessible extracellularly. The experiment docking $\mathrm{MPP}^{+}$to the outward-open cleft is consistent with the interpretation that the high-affinity and the two low-affinity $\mathrm{MPP}^{+}$binding sites are located within the outward-open cleft.

In summary, the data described in this paper provide a rationale for improving in vitro and in silico testing of NMEs for interaction with OCTs. From the demonstration of three different binding sites for one substrate in one rOCT1 monomer, in combination with the property of OCTs to translocate structurally diverse compounds, we conclude that these highly polyspecific transporters contain various binding sites that 


\section{outward open, from outside}
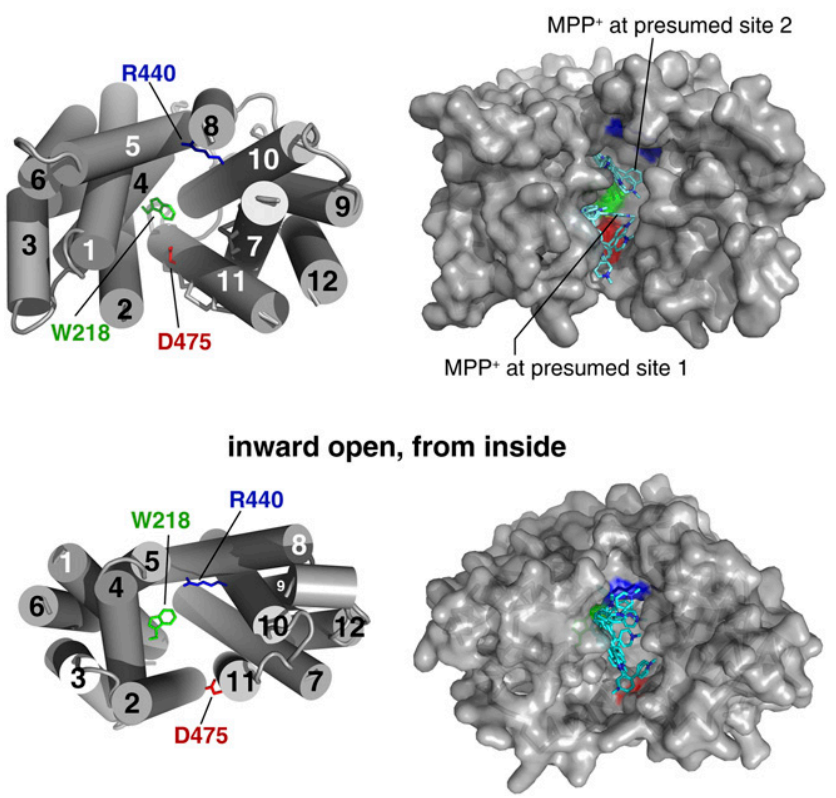

Fig. 9. Docking of $\mathrm{MPP}^{+}$to the modeled outward-open and inward-open binding cleft of rOCT1. Docking was performed to previously built 3D homology models of rOCT1. Docking of $\mathrm{MPP}^{+}$molecules was restrained to occur within spheres of $7 \AA$ around Trp218, Arg440, and Asp475. Thirty $\mathrm{MPP}^{+}$molecules with the highest docking energies for each transporter conformation were selected. Twenty-six and 22 molecules were found to be located inside the outward-open and inward-open cleft, respectively. Owing to highly similar docking positions, not all $\mathrm{MPP}^{+}$moieties are shown in the presentations.

are mostly or exclusively located within large clefts that may have both extracellular or intracellular exposure. Future attempts to crystallize transporters of the SLC22 family may be successful if performed in the presence of structurally different ligands and lipids containing unsaturated fatty acids and/or cholesterol. The demonstration of one high-affinity and two low-affinity binding sites in rOCT1 and the identification of amino acids that participate in formation of the low-affinity binding sites will help to interpret the crystal structures obtained.

\section{Acknowledgments}

We thank Martin Lohse (Institute of Pharmacology and Toxicology, University Würzburg) for providing laboratory space and giving technical support and Michael Christof (Institute of Anatomy and Cell Biology, University Würzburg) for preparing the figures.

\section{Authorship Contributions}

Participated in research design: Keller, Mueller, Bernhard, Koepsell.

Conducted experiments: Keller, Gorboulev, Mueller, Bernhard.

Contributed new reagents or analytic tools: Bernhard, Dötsch.

Performed data analysis: Keller, Mueller, Koepsell.

Wrote or contributed to the writing of the manuscript: Koepsell.

\section{References}

Ahlin G, Chen L, Lazorova L, Chen Y, Ianculescu AG, Davis RL, Giacomini KM, and Artursson P (2011) Genotype-dependent effects of inhibitors of the organic cation transporter, OCT1: predictions of metformin interactions. Pharmacogenomics $J$ 11:400-411.

Ahlin G, Karlsson J, Pedersen JM, Gustavsson L, Larsson R, Matsson P, Norinder U, Bergström CAS, and Artursson P (2008) Structural requirements for drug inhibition of the liver specific human organic cation transport protein 1. J Med Chem 51:5932-5942.
Amphoux A, Vialou V, Drescher E, Brüss M, Mannoury La Cour C, Rochat C, Millan MJ, Giros B, Bönisch H, and Gautron S (2006) Differential pharmacological in vitro properties of organic cation transporters and regional distribution in rat brain. Neuropharmacology 50:941-952.

Belzer M, Morales M, Jagadish B, Mash EA, and Wright SH (2013) Substratedependent ligand inhibition of the human organic cation transporter OCT2. $J$ Pharmacol Exp Ther 346:300-310.

Brast S, Grabner A, Sucic S, Sitte HH, Hermann E, Pavenstädt H, Schlatter E, and Ciarimboli G (2012) The cysteines of the extracellular loop are crucial for trafficking of human organic cation transporter 2 to the plasma membrane and are involved in oligomerization. FASEB $J$ 26:976-986.

Budiman T, Bamberg E, Koepsell H, and Nagel G (2000) Mechanism of electrogenic cation transport by the cloned organic cation transporter 2 from rat. J Biol Chem 275:29413-29420.

Busch AE, Quester S, Ulzheimer JC, Waldegger S, Gorboulev V, Arndt P, Lang F, and Koepsell H (1996) Electrogenic properties and substrate specificity of the polyspecific rat cation transporter rOCT1. J Biol Chem 271:32599-32604.

Chen EC, Khuri N, Liang X, Stecula A, Chien HC, Yee SW, Huang Y, Sali A, and Giacomini KM (2017) Discovery of competitive and noncompetitive ligands of the organic cation transporter 1 (OCT1; SLC22A1). J Med Chem 60:2685-2696.

Dakal TC, Kumar R, and Ramotar D (2017) Structural modeling of human organic cation transporters. Comput Biol Chem 68:153-163.

Denisov IG, Grinkova YV, Lazarides AA, and Sligar SG (2004) Directed self-assembly of monodisperse phospholipid bilayer Nanodiscs with controlled size. J Am Chem Soc 126:3477-3487.

Egenberger B, Gorboulev V, Keller T, Gorbunov D, Gottlieb N, Geiger D, Mueller TD, and Koepsell H (2012) A substrate binding hinge domain is critical for transportrelated structural changes of organic cation transporter 1. J Biol Chem 287: 31561-31573.

Giacomini KM, Huang S-M, Tweedie DJ, Benet LZ, Brouwer KL, Chu X, Dahlin A, Evers R, Fischer V, Hillgren KM, et al.; International Transporter Consortium (2010) Membrane transporters in drug development. Nat Rev Drug Discov 9: $215-236$.

Gorboulev V, Rehman S, Albert CM, Roth U, Meyer MJ, Tzvetkov MV, Mueller TD, and Koepsell H (2018) Assay conditions influence affinities of rat organic cation transporter 1: analysis of mutagenesis in the modeled outward-facing cleft by measuring effects of substrates and inhibitors on initial uptake. Mol Pharmacol $\mathbf{9 3}$ : $402-415$.

Gorboulev V, Shatskaya N, Volk C, and Koepsell H (2005) Subtype-specific affinity for corticosterone of rat organic cation transporters rOCT1 and rOCT2 depends on three amino acids within the substrate binding region. Mol Pharmacol 67: $1612-1619$.

Gorboulev V, Volk C, Arndt P, Akhoundova A, and Koepsell H (1999) Selectivity of the polyspecific cation transporter rOCT1 is changed by mutation of aspartate 475 to glutamate. Mol Pharmacol 56:1254-1261.

Gorbunov D, Gorboulev V, Shatskaya N, Mueller T, Bamberg E, Friedrich T, and Koepsell H (2008) High-affinity cation binding to organic cation transporter 1 induces movement of helix 11 and blocks transport after mutations in a modeled interaction domain between two helices. Mol Pharmacol 73:50-61

Holyoake J and Sansom MSP (2007) Conformational change in an MFS protein: MD simulations of LacY. Structure 15:873-884.

Kaback HR, Dunten R, Frillingos S, Venkatesan P, Kwaw I, Zhang W, and Ermolova N (2007) Site-directed alkylation and the alternating access model for LacY. Proc Natl Acad Sci USA 104:491-494.

Keller T, Egenberger B, Gorboulev V, Bernhard F, Uzelac Z, Gorbunov D, Wirth C, Koppatz S, Dötsch V, Hunte C, et al. (2011) The large extracellular loop of organic cation transporter 1 influences substrate affinity and is pivotal for oligomerization. J Biol Chem 286:37874-37886.

Keller T, Elfeber M, Gorboulev V, Reiländer H, and Koepsell H (2005) Purification and functional reconstitution of the rat organic cation transporter OCT1. Biochemistry 44:12253-12263.

Keller T, Schwarz D, Bernhard F, Dötsch V, Hunte C, Gorboulev V, and Koepsell H (2008) Cell free expression and functional reconstitution of eukaryotic drug transporters. Biochemistry 47:4552-4564

Klammt C, Löhr F, Schäfer B, Haase W, Dötsch V, Rüterjans H, Glaubitz C, and Bernhard F (2004) High level cell-free expression and specific labeling of integral membrane proteins. Eur J Biochem 271:568-580.

Koepsell H (2013) The SLC22 family with transporters of organic cations, anions and zwitterions. Mol Aspects Med 34:413-435.

Koepsell H (2018) Multiple binding sites in organic cation transporters require sophisticated procedures to identify interactions of novel drugs. Biol Chem DOI: 10.1515/hsz-2018-0191 [published ahead of print].

Koepsell H, Lips K, and Volk C (2007) Polyspecific organic cation transporters: structure, function, physiological roles, and biopharmaceutical implications. Pharm Res 24:1227-1251.

Laganowsky A, Reading E, Allison TM, Ulmschneider MB, Degiacomi MT, Baldwin AJ, and Robinson CV (2014) Membrane proteins bind lipids selectively to modulate their structure and function. Nature 510:172-175.

Martinez Molledo M, Quistgaard EM, Flayhan A, Pieprzyk J, and Löw C (2018) Multispecific substrate recognition in a proton-dependent oligopeptide transporter. Structure 26:467-476.e4.

Meyer-Wentrup F, Karbach U, Gorboulev V, Arndt P, and Koepsell H (1998) Membrane localization of the electrogenic cation transporter rOCT1 in rat liver. Biochem Biophys Res Commun 248:673-678.

Minuesa G, Albert C, Pastor-Anglada M, Martinez-Picado J, and Koepsell H (2017) Response to "Tenofovir disoproxil fumarate is not an inhibitor of human organic cation transporter 1". J Pharmacol Exp Ther 360:343-345.

Minuesa G, Huber-Ruano I, Pastor-Anglada M, Koepsell H, Clotet B, and MartinezPicado J (2011) Drug uptake transporters in antiretroviral therapy. Pharmacol Ther 132:268-279. 
Minuesa G, Volk C, Molina-Arcas M, Gorboulev V, Erkizia I, Arndt P, Clotet B, Pastor-Anglada M, Koepsell H, and Martinez-Picado J (2009) Transport of lamivudine [(-)-beta-L-2',3'-dideoxy-3'-thiacytidine] and high-affinity interaction of nucleoside reverse transcriptase inhibitors with human organic cation transporters 1, 2, and 3. J Pharmacol Exp Ther 329:252-261.

Nies AT, Koepsell H, Damme K, and Schwab M (2011) Organic cation transporters (OCTs, MATEs), in vitro and in vivo evidence for the importance in drug therapy. Handb Exp Pharmacol 201:105-167.

Popp C, Gorboulev V, Müller TD, Gorbunov D, Shatskaya N, and Koepsell H (2005) Amino acids critical for substrate affinity of rat organic cation transporter 1 line the substrate binding region in a model derived from the tertiary structure of lactose permease. Mol Pharmacol 67:1600-1611.

Roos C, Kai L, Proverbio D, Ghoshdastider U, Filipek S, Dötsch V, and Bernhard F (2013) Co-translational association of cell-free expressed membrane proteins with supplied lipid bilayers. Mol Membr Biol 30:75-89.

Roos C, Zocher M, Müller D, Münch D, Schneider T, Sahl HG, Scholz F, Wacht veitl J, Ma Y, Proverbio D, et al. (2012) Characterization of co-translationally formed nanodisc complexes with small multidrug transporters, proteorhodopsin and with the E. coli MraY translocase. Biochim Biophys Acta 1818 3098-3106.

Sandoval PJ, Zorn KM, Clark AM, Ekins S, and Wright SH (2018) Assessment of substrate-dependent ligand interactions at the organic cation transporter OCT2 using six model substrates. Mol Pharmacol 94:1057-1068.

Schmitt BM and Koepsell H (2005) Alkali cation binding and permeation in the rat organic cation transporter rOCT2. J Biol Chem 280:24481-24490.

Thévenod F, Ciarimboli G, Leistner M, Wolff NA, Lee WK, Schatz I, Keller T, AlMonajjed R, Gorboulev V, and Koepsell H (2013) Substrate- and cell contactdependent inhibitor affinity of human organic cation transporter 2: studies with two classical organic cation substrates and the novel substrate $\mathrm{cd}^{2+} \cdot$ Mol Pharm 10:3045-3056.

U.S. Food and Drug Administration (2012) Guidance for Industry: Drug Interaction Studies - Study Design, Data Analysis, Implications for Dosing, and Labeling Recommendations (Huang SM and Zhang L eds) pp 1-79, Bethesda, MD

U.S. Food and Drug Adminstration (2017) Guidance for industry: In vitro metabolismand transporter-mediated drug-drug interaction studies. pp 1-47, Bethesda, MD.

Volk C, Gorboulev V, Kotzsch A, Müller TD, and Koepsell H (2009) Five amino acids in the innermost cavity of the substrate binding cleft of organic cation transporter 1 interact with extracellular and intracellular corticosterone. Mol Pharmacol 76: 275-289.

Yin J, Duan H, and Wang J (2016) Impact of substrate-dependent inhibition on renal organic cation transporters hOCT2 and hMATE1/2-K-mediated drug transport and intracellular accumulation. J Pharmacol Exp Ther 359:401-410.

Zacharias DA, Violin JD, Newton AC, and Tsien RY (2002) Partitioning of lipidmodified monomeric GFPs into membrane microdomains of live cells. Science $\mathbf{2 9 6}$ 913-916.

Zamek-Gliszczynski MJ, Giacomini KM, and Zhang L (2018) Emerging clinical importance of hepatic organic cation transporter 1 (OCT1) in drug pharmacokinetics, dynamics, pharmacogenetic variability, and drug interactions. Clin Pharmacol Ther 103:758-760.

Address correspondence to: Dr. Hermann Koepsell, Department of Molecular Plant Physiology and Biophysics, Julius-von-Sachs-Institute, Julius-von-Sachs-Platz 2, 97082 Würzburg, Germany. E-mail: Hermann@ Koepsell.de 